\title{
Two-layer nanofluid flow and heat transfer in a horizontal microchannel with electric double layer effects and magnetic field
}

Qingkai Zhao, Hang Xu, Longbin Tao

\begin{abstract}
Purpose - The purpose of paper is to investigate the immiscible two-layer heat fluid flows in the presence of the electric double layer (EDL) and magnetic field. The effects of EDL, magnetic field and the viscous dissipative term on fluid velocity and temperature, as well as the important physical quantities are examined and discussed.

Design/methodology/approach - The two regions in a horizontal microchannel with one layer being filled with a nanofluid and the other with a viscous Newtonian fluid. The nanofluid flow in the lower layer is described by the Buongiorno's nanofluid model with passively-controlled model at the boundaries. An appropriate set of non-dimensional quantities are employed to simplify the nonlinear systems. The resulting coupled nonlinear equations are solved by homotopy analysis method.

Findings - The present work demonstrates that increasing the EDL thickness and Hartmann number can restrain the fluid flow. The Brinkmann number has a significant role in the enhancement of heat transfer. It is also identified that the influence of EDL effects on micro flow cannot be ignored.

Originality/Value - The effects of viscous dissipation involved in heat transfer process and the body force due to the EDL and the magnetic field are considered in the thermal energy and momentum equations for both regions, respectively. The detailed derivation procedure of the analytical solution for electrostatic potential are provided. The analytical solutions can lead to improved understanding of the complex microfluidic systems.
\end{abstract}

Keywords Two-layer flow; Nanofluid; Electric double layer; Magnetic field; Passively-controlled model; Homotopy analysis method

Paper type - Research paper

Nomenclature

\begin{tabular}{|ll|}
\hline $\mathrm{Br}_{1}, \mathrm{Br}_{2}$ & Brinkman number \\
$B_{0}$ & magnetic flux density in $y$-direction \\
$c_{p 1}, c_{p 2}$ & magnetic flux density vector \\
$C$ & npecific heat capacities at constant pressure $\left[\mathrm{J} \mathrm{kg}^{-1} \mathrm{~K}^{-1}\right]$ \\
$C_{0}$ & reference nanoparticle volume fraction \\
$C_{1}$ & nanoparticle volume fraction at the interface \\
\hline
\end{tabular}




\begin{tabular}{|c|c|}
\hline$C_{f 1}, C_{f 2}$ & local skin friction coefficients on the microchannel walls $\left[\mathrm{kg} \mathrm{m}^{-3}\right]$ \\
\hline$D_{B}$ & Brownian diffusion coefficient $\left[\mathrm{m}^{2} \mathrm{~s}^{-1}\right]$ \\
\hline$D_{\mathrm{T}}$ & thermophoretic diffusion coefficient $\left[\mathrm{m}^{2} \mathrm{~s}^{-1}\right]$ \\
\hline & charge of a proton $[\mathrm{C}]$ \\
\hline$E(m)$ & $\begin{array}{l}\text { maximum total average squared residual error for homotopy analysis } \\
\text { method computation order } m\end{array}$ \\
\hline$E c_{1}, E c_{2}$ & Eckert number \\
\hline$E_{s 1}, E_{s 2}$ & streaming potentials $[\mathrm{V}]$ \\
\hline $\bar{E}_{s 1}, \bar{E}_{s 2}$ & dimensionless streaming potentials \\
\hline$E_{x 1}, E_{x 2}$ & electric field strengths $\left[\mathrm{V} \mathrm{m}^{-1}\right.$ or $\mathrm{N} \mathrm{C}^{-1}$ ] \\
\hline $\mathbf{F}_{1}, \mathbf{F}_{2}$ & electrical body forces $\left[\mathrm{N} \mathrm{m}^{-3}\right]$ \\
\hline$g$ & gravitational acceleration $\left[\mathrm{m} \mathrm{s}^{-2}\right]$ \\
\hline$G_{1}, G_{2}$ & dimensionless quantity, represent the ratio of EDL force to viscous force \\
\hline$G_{3}, G_{4}$ & $\begin{array}{l}\text { dimensionless quantity, represent the ratio of streaming current to } \\
\text { conduction current }\end{array}$ \\
\hline$h_{1}, h_{2}$ & the hight of two-layer fluid in regions I and II, respectively [m] \\
\hline$H a_{1}, H a_{2}$ & Hartmann number \\
\hline$I_{c 1}, I_{c 2}$ & conduction currents $[\mathrm{A}]$ \\
\hline$I_{s 1}, I_{s 2}$ & streaming currents $[\mathrm{A}]$ \\
\hline $\mathbf{J}_{1}, \mathbf{J}_{2}$ & current density vectors \\
\hline$k_{1}, k_{2}$ & Debye-Hückel parameters $\left[\mathrm{m}^{-1}\right]$ \\
\hline$k_{b}$ & Boltzmann constant $\left[\mathrm{J} \mathrm{mol}^{-1} \mathrm{~K}^{-1}\right]$ \\
\hline$k_{f 1}, k_{f 2}$ & thermal conductivities of the liquids $\left[\mathrm{W} \mathrm{m} \mathrm{m}^{-1} \mathrm{~K}^{-1}\right.$ ] \\
\hline$L$ & length of the microchannel $[\mathrm{m}]$ \\
\hline
\end{tabular}




\begin{tabular}{|c|c|}
\hline$n_{i}$ & ionic number concentration of the $i$ th species \\
\hline$n_{01}, n_{02}$ & the bulk ionic concentrations $\left[\mathrm{m}^{-3}\right]$ \\
\hline $\mathrm{Nb}$ & Brownian motion parameter \\
\hline$N t$ & thermophoresis parameter \\
\hline$N u_{1}, N u_{2}$ & local Nusselt number \\
\hline$p_{1}, p_{2}$ & pressures $[\mathrm{Pa}]$ \\
\hline$P_{1}, P_{2}$ & non-dimensional pressure gradient parameters \\
\hline$P r_{1}, P r_{2}$ & Prandtl number \\
\hline$q_{w 1}, q_{w 2}$ & wall heat fluxes on the two wall of the microchannel $\left[\mathrm{W} \mathrm{m}^{-2}\right]$ \\
\hline$q_{C}$ & local wall flux of nanoparticles on the lower wall of the microchannel \\
\hline & {$\left[\mathrm{kg} \mathrm{m}^{-2} \mathrm{~s}^{-1}\right]$} \\
\hline $\mathrm{Re}_{1}, \mathrm{Re}_{2}$ & Reynolds number \\
\hline$T_{1}, T_{2}$ & temperatures $[\mathrm{K}]$ \\
\hline$T_{0}$ & reference temperature $[\mathrm{K}]$ \\
\hline$T_{w 1}, T_{w 2}$ & temperatures on the microchannel wall surface $[\mathrm{K}]$ \\
\hline$\hat{T}$ & absolute temperature $[\mathrm{K}]$ \\
\hline$u_{1}, u_{2}$ & $\mathrm{x}$-component of the fluid velocities $\left[\mathrm{m} \mathrm{s}^{-1}\right]$ \\
\hline$u_{m}$ & average velocity at the entrance $\left[\mathrm{m} \mathrm{s}^{-1}\right]$ \\
\hline$U_{1}, U_{2}$ & dimensionless velocities of the fluid \\
\hline $\mathbf{V}_{1}, \mathbf{V}_{2}$ & velocity vectors \\
\hline$W$ & width of the microchannel $[\mathrm{m}]$ \\
\hline$x, y$ & Cartesian coordinates \\
\hline Y & non-dimensional Cartesian coordinate \\
\hline
\end{tabular}




$$
\hat{z}_{1}, \hat{z}_{2} \quad \text { the valences of ions }
$$

\section{Greek symbols}

$\alpha_{1}, \alpha_{2} \quad$ thermal diffusivities of the nanofluid $\left[\mathrm{m}^{2} \mathrm{~s}^{-1}\right]$

$\varepsilon_{0}$

permittivity of vacuum $\left[\mathrm{C} \mathrm{V}^{-1} \mathrm{~m}^{-1}\right]$

$\varepsilon_{1}, \varepsilon_{2} \quad$ dielectric constants of the medium

$\kappa_{1}, \kappa_{2} \quad$ parameters characterizing the EDL

$\lambda_{01}, \lambda_{02} \quad$ electrical conductivities of the fluid $\left[\Omega^{-1} \mathrm{~m}^{-1}\right]$

$\mu_{1}, \mu_{2} \quad$ dynamic viscosities of the fluid $\left[\mathrm{kg} \mathrm{m}^{-1} \mathrm{~s}^{-1}\right]$

$v_{1}, v_{2} \quad$ kinematic viscosities of the fluid $\left[\mathrm{m}^{2} \mathrm{~s}^{-1}\right]$

$\sigma_{1}, \sigma_{2} \quad$ electrical conductivities

$\theta_{1}, \theta_{2} \quad$ non-dimensional temperature distributions

$\phi \quad$ non-dimensional nanoparticle volume fraction

$\Phi_{1}, \Phi_{2} \quad$ viscous dissipation terms

$\rho_{e 1}, \rho_{e 2} \quad$ charge densities $\left[\mathrm{C} \mathrm{m}^{-3}\right]$

$\rho_{1}, \rho_{2} \quad$ density of the fluids $\left[\mathrm{kg} \mathrm{m}^{-3}\right]$

$\tau \quad$ heat capacity ratio

$\tau_{w 1}, \tau_{w 2} \quad$ shear stresses on the wall of the microchannel $[\mathrm{Pa}]$

$\zeta_{1}, \zeta_{2} \quad$ zeta potentials [V]

$\psi_{1}, \psi_{2} \quad$ electrostatic potentials [V]

$\Psi_{1}, \Psi_{2} \quad$ dimensionless electrostatic potentials

$\Omega_{1}, \Omega_{2} \quad$ cross-sectional areas $\left[\mathrm{m}^{2}\right]$

Subscript 
physical quantities on the microchannel wall

denote the fluid and solid particles

1,2

Denote the quantities for regions I and II, respectively

\section{Introduction}

Recently, flow and heat transfer in a microchannel has gained great interest due to its widely practical and potential applications in many industrial fields, such as cooling of micro-electromechanical system(MEMS), production of a penny-sized nuclear battery for small and microelectronic systems, enhancement of heat transfer in aerospace technology (Darabi and Ekula, 2003; Donaldson, 2009). Mathematical approaches for fluid and heat transfer problems have also been developed (Lewis et al., 1996; Kulasegaram et al., 2004; Lewis et al., 2004; Nithiarasu et al., 2016). However, some experimental observations and theoretical studies (Wang and Peng, 1994; Mala et al., 1997; Mala and Li, 1999; Guo and Li, 2003) showed behaviour of thermal fluid flow in a microchannel is different from that in macroscopic scale. Wang and Peng (1994) noticed that the transition and laminar heat transfer behaviour in microchannel are very complicated, and the behaviour may be strongly affected by liquid temperature, velocity and microchannel size. Mala et al. (1997) and Mala and Li (1999) indicated that the fluid flow and heat transfer in a microchannel could be significantly affected by some interfacial factors such as EDL (Hunter, 1981). It is widely accepted that the effect of the EDL on velocity distribution and heat transfer cannot be neglected in microchannel fluid flow. Microfluidic is one of the most important research fields in MEMS, and microchannels are critical part of fluidic-MEMS. When the fluid flows through a microchannel, the EDL can be formed due to hydrolysis and adsorption. The effects of EDL plays an important role in transport processes in microchannel. For instance, the EDL can restrain the fluid motion which can further affect the pressure, heat transfer and the distribution of nanoparticles. Some studies on fluid flow in microchannel actuated by the application of electrokinetic force under different situations. Ren et al. (2001) examined the magnitude of the additional flow resistance 
caused by the electrokinetic effect and pointed out that the EDL effect is the primary cause of the significantly higher-pressure drop for pure water and dilute aqueous ionic solutions flowing through microchannels. Ren and Li (2005) further developed a theoretical model of the EDL field to investigate the effects of electric double layer on pressure-driven flow in microchannel. You and Gu (2010) studied the effects of EDL on the velocity distribution and the flow stability of the developed laminar flow in microchannels. Recently, Jing et al. (2017) investigated fully developed pressuredriven flow in a microchannel with consideration of the combined effect of surface charge-induced EDL and surface charge-dependent slip. Based on their studies, the authors revealed that EDL is a key factor to affect the fluidic behaviour and the thermal performances of pressure-driven flow, including viscous dissipation and convective heat transfer (Jing, 2017; Srinivas, 2016; Qi and Ng, 2018; Zheng and Jian, 2018).

As a new kind of heat transfer medium, nanofluids have attracted much attention of researchers in various fields. One of the most important features of nanofluids is their capability to enhance heat transfer performance of the base fluid (Choi, 1995). Many researchers have devoted to study the flow and heat transfer mechanism of nanofluid and confirmed that the suspended nanoparticles significantly improve heat transfer process primarily owning to the nanofluid possessing larger heat transfer coefficient than that of the pure fluid (Xuan and Li, 2003; Heris et al., 2006; Williams et al., 2008; Kuznetsov and Nield, 2010; Wang et al., 2014; Manikandan and Rajan, 2015; Karimipour et al., 2015). On the other hand, the subject of multi-fluid flow and heat transfer has been studied extensively due to its importance in petroleum industry, plasma physics and chemical industry. Many scientific and technological applications involve multi-phase flow and multi-layer flow (Lewis and Ghafouri, 1997; Redapangu et al., 2012; Li et al., 2010; Moh'd and Al-Dafaie, 2014), such as electroosmotic pumps consist of two immiscible or miscible liquids with different viscosities. Previously, Arco et al. (1991) studied the thermocapillary convection in a two layer fluid system with flat interface. Subsequently, the immiscible two layer fluid system with flat interface system has been investigated in recent years. Xie and Jian (2017) studied the two immiscible fluids electroosmotic flow in the presence of magnetic field through a microparallel channel. The effects of magnetic field parameters on the local and total 
entropy generation rates are studied in their work. Shit et al. (2016) investigated a twolayer fluid flow and heat transfer in a hydrophobic micro-channel by considering the combined influence of pressure gradient and electro-osmotic forces and demonstrated that the zeta potential difference plays an important role in controlling fluid velocity in the micro-channel. Elmaboud et al. (2019) studied the electromagnetic flow for twolayer of immiscible fluids in an inclined channel and applied the homotopy analysis method in the solution process, and revealed that the velocity decelerates with increasing the magnetic field due to the Lorentz force.

More recently, the Buongiorno's model (Buongiorno, 2006) has often been adopted by researchers to study the flow and heat transfer of nanofluids in various physical situations. Rohni et al. (2013) investigated numerically the unsteady nanofluid flow over a continuously shrinking surface with wall mass suction. Sheremet and Pop (2014) studied the laminar natural convective nanofluid flow and heat transfer in a square porous cavity with sinusoidal temperature distributions on both side walls. Rahman et al. (2015) studied the steady nanofluid flow past a permeable exponentially shrinking surface by numerical simulation. Yu et al. (2018) investigated the laminar mixed convection nanofluid flow in an inclined liddriven cavity and applied Buongiorno's nanofluid model to describe the nanofluid behaviours. Their works not only demonstrated that the nanofluid can enhance heat transfer, but also confirmed the validity of the Buongiorno's model.

In this paper, the steady immiscible two-layer flow in a horizontal microchannel with the EDL effects and magnetic field is studied. The body forces caused by the EDL and magnetic field are considered in the momentum equation for both regions. The Buongiorno's model is adopted due to its advantage in explaining well the relationship between the nanoparticles and the base liquid (Zhao et al., 2018). The energy and the concentration of the nanoparticle equations are established respectively. As one of the highlights of the present study, a passively controlled nanofluid model was adopted at the lower plate (Kuznetsov and Nield, 2013). Through nondimensionalization, the governing equations are transformed into nonlinear ordinary differential equations. The resulting coupled nonlinear equations are solved by homotopy analysis method (HAM) (Liao, 2012), and the analytical solutions for electric potential, velocity, temperature 
and nanoparticle concentration are obtained. The effects of EDL, Hartmann number, Brinkman number and thermophoresis parameter on various physical quantities are examined and discussed. The improved understanding of these fundamental phenomena and their mechanisms is deemed crucial for to the optimal design of micro/nanofluidic systems.

\section{Physical and mathematical modelling}

In the following sections, the problem of a nanofluid flow adjacent to a clear fluid through a microchannel between two parallel plates with the effects of EDL and magnetic field is discussed in detail. The region I $\left(0<y<h_{1}\right)$ is filled with a fluid of thermal diffusivity $\alpha_{1}$, viscosity $\mu_{1}$ and density $\rho_{1}$. The region II $\left(-h_{2}<y<0\right)$ is filled with a nanofluid, the density, viscosity and thermal diffusivity are $\rho_{2}, \mu_{2}$, and $\alpha_{2}$, respectively. The boundary walls of the channel are isothermal and are held at different temperatures. The temperature of the upper wall and the lower wall are $T_{w 1}$ and $T_{w 2}$, respectively. The nanoparticle volume fraction at the interface is $C_{1}$, while a passively controlled nanofluid model is employed on the lower wall. The magnetic flux density $\mathbf{B}$ is imposed along the y-axis. The length of the channel is L as illustrated in Fig.1.

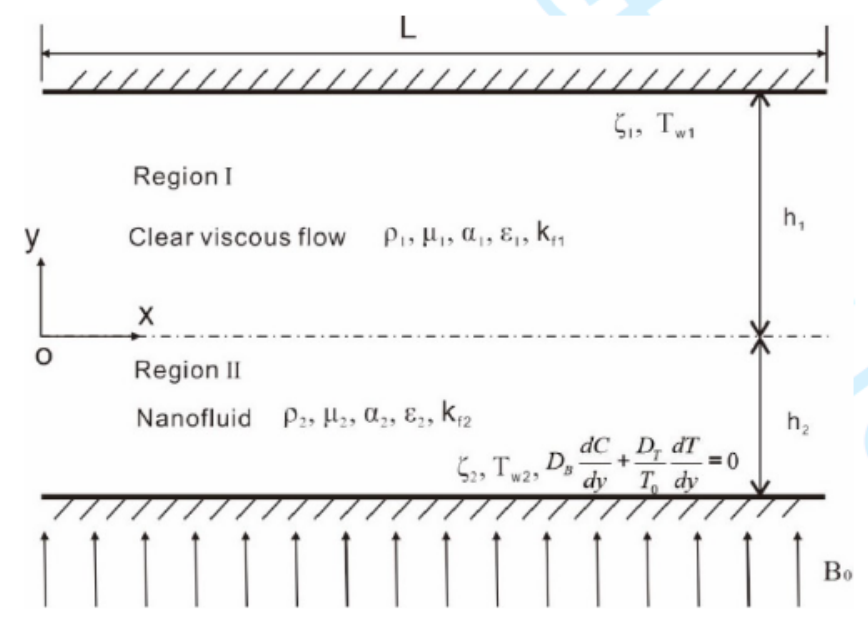

Fig1. Schematic of the physical model

The flow is assumed to be steady, laminar, fully developed in both regions and the thermophysical properties of the fluid remain constant. The pressure gradients are assumed to be constants in both regions. The nanofluid flow in the lower layer is described by the Buongiorno's nanofluid model. Based on the previous work (Shit et 
al., 2016; Xie and Jian, 2017), the physical model and boundary conditions in this research are valid. Therefore, the governing equations of the problem in both regions are the electrostatic potential equation, the conservations of the total mass, momentum, thermal energy equation and nanoparticle volume fraction equation and can be written as

i). Region I

$$
\begin{gathered}
\nabla^{2} \psi_{1}=-\frac{\rho_{e 1}}{\varepsilon_{0} \varepsilon_{1}}, \\
\nabla \cdot \mathbf{V}_{1}=0 \\
\rho_{1}\left(\mathbf{V}_{1} \cdot \nabla\right) \mathbf{V}_{1}=-\nabla p_{1}+\mu_{1} \nabla^{2} \mathbf{V}_{1}+\mathbf{F}_{1}, \\
\left(\mathbf{V}_{1} \cdot \nabla\right) T_{1}=\alpha_{1} \nabla^{2} T_{1}+\frac{\mu_{1}}{\rho_{1} c_{p 1}} \Phi_{1}
\end{gathered}
$$

ii). Region II

$$
\begin{gathered}
\nabla^{2} \psi_{2}=-\frac{\rho_{e 2}}{\varepsilon_{0} \varepsilon_{2}}, \\
\nabla \cdot \mathbf{V}_{2}=0 \\
\rho_{2}\left(\mathbf{V}_{2} \cdot \nabla\right) \mathbf{V}_{2}=-\nabla p_{2}+\mu_{2} \nabla^{2} \mathbf{V}_{2}+\mathbf{F}_{2}, \\
\left(\mathbf{V}_{2} \cdot \nabla\right) T_{2}=\alpha_{2} \nabla^{2} T_{2}+\tau\left[D_{B} \nabla T_{2} \cdot \nabla C+\frac{D_{T}}{T_{0}} \nabla T_{2} \cdot \nabla T_{2}\right]+\frac{\mu_{2}}{\rho_{2} c_{p 2}} \Phi_{2}, \\
\left(\mathbf{V}_{2} \cdot \nabla\right) C=D_{B} \nabla^{2} C+\frac{D_{T}}{T_{0}} \nabla^{2} T_{2},
\end{gathered}
$$

In the above equations, the subscripts $i=1,2$ denote the values for regions I and II, respectively. Here, $\psi_{i}$ are the electrostatic potentials in the electric double layer, $\varepsilon_{i}$ are the dielectric constants of the fluid medium, $\varepsilon_{0}$ is the permittivity of vacuum, $\mathbf{V}_{i}$ are the velocity vectors, $p_{i}$ are the pressures. The vector $\mathbf{F}_{i}=\mathbf{E}_{x i} \rho_{e i}+\mathbf{J}_{i} \times \mathbf{B}$ are the electrical body forces originating from the presence of the electric double layer and magnetic field, $\mathbf{E}_{x i}$ are the electric field strengths. $\rho_{e i}$ are the charge densities, $\mathbf{J}_{i}$ are the current density vectors, $\mathbf{B}=\left(0, B_{0}\right)$ is the magnetic flux density vector, $T_{i}$ and $T_{0}$ denote the temperatures and the reference temperature, respectively. $C$ is the 
nanoparticle volume fraction, $\Phi_{i}$ are the viscous dissipation terms, $c_{p i}$ are the heat capacities. $D_{B}$ and $D_{T}$ represent the Brownian diffusion coefficient and the thermophoretic diffusion coefficient respectively, and $\tau=\left(\rho_{2} c_{p 2}\right)_{p} /\left(\rho_{2} c_{p 2}\right)_{f}$ is the heat capacity ratio. Subscripts $p$ and $f$ denote the nanoparticles and the base fluid in region II, respectively.

For a parallel flow in channels, assume, without loss of generality, the flow is unidirectional so that the velocity component is equal to zero along $y$-direction. Based on these assumptions, the governing equations (1)-(9) are reduce to

i). Region I

$$
\begin{gathered}
\frac{d^{2} \psi_{1}}{d y^{2}}=-\frac{\rho_{e 1}}{\varepsilon_{0} \varepsilon_{1}} \\
\mu_{1} \frac{d^{2} u_{1}}{d y^{2}}-\frac{d p_{1}}{d x}-\sigma_{1} B_{0}^{2} u_{1}+E_{x 1} \rho_{e_{1}}=0 \\
\alpha_{1} \frac{d^{2} T_{1}}{d y^{2}}+\frac{\mu_{1}}{\rho_{1} c_{p 1}}\left(\frac{d u_{1}}{d y}\right)^{2}=0
\end{gathered}
$$

ii). Region II

$$
\begin{gathered}
\frac{d^{2} \psi_{2}}{d y^{2}}=-\frac{\rho_{e 2}}{\varepsilon_{0} \varepsilon_{2}}, \\
\mu_{2} \frac{d^{2} u_{2}}{d y^{2}}-\frac{d p_{2}}{d x}-\sigma_{2} B_{0}^{2} u_{2}+E_{x 2} \rho_{e 2}=0, \\
\alpha_{2} \frac{d^{2} T_{2}}{d y^{2}}+\tau\left[D_{B} \frac{d T_{2}}{d y} \frac{d C}{d y}+\frac{D_{T}}{T_{0}}\left(\frac{d T_{2}}{d y}\right)^{2}\right]+\frac{\mu_{2}}{\rho_{2} c_{p 2}}\left(\frac{d u_{2}}{d y}\right)^{2}=0, \\
D_{B} \frac{d^{2} C}{d y^{2}}+\frac{D_{T}}{T_{0}} \frac{d^{2} T_{2}}{d y^{2}}=0,
\end{gathered}
$$

where, $u_{i}$ and $\sigma_{i}(i=1,2)$ are the fluid velocities along the x-axis and the electrical conductivities in the region I and region II, respectively.

It is assumed that the electrical potential, the velocity, the shear stress, the temperature, and the flux at the interface are continuous. The velocities of fluid in both regions satisfy the non-slip condition on the wall surface. The boundary conditions for the temperature are isothermal. Based on previous work (Kuznetsov and Nield, 2013), 
the nanoparticle volume fraction satisfies a passively controlled nanofluid model at the boundary. Therefore, the appropriate boundary and interface conditions of Eqs.(10)-(16) are

$$
\begin{aligned}
& \left.\begin{array}{l}
\psi_{1}(y)=\zeta_{1}, u_{1}(y)=0, T_{1}(y)=T_{w 1}, \quad \text { at } y=h_{1} . \\
\psi_{1}(y)=\psi_{2}(y), u_{1}(y)=u_{2}(y), T_{1}(y)=T_{2}(y), C(y)=C_{1}, \\
\varepsilon_{1} \frac{d \psi_{1}}{d y}=\varepsilon_{2} \frac{d \psi_{2}}{d y}, \mu_{1} \frac{d u_{1}}{d y}=\mu_{2} \frac{d u_{2}}{d y}, k_{f 1} \frac{d T_{1}}{d y}=k_{f 2} \frac{d T_{2}}{d y},
\end{array}\right\} \text { at } y=0 . \\
& \psi_{2}(y)=\zeta_{2}, u_{2}(y)=0, T_{2}(y)=T_{w 2}, D_{B} \frac{d C}{d y}+\frac{D_{T}}{T_{0}} \frac{d T_{2}}{d y}=0, \text { at } y=-h_{2} .
\end{aligned}
$$

where $\zeta_{i}$ and $k_{f i}(i=1,2)$ are the zeta potentials and the thermal conductivities in the region I and region II, respectively.

\subsection{The electrostatic potential equation and the analytical solution}

Consider a microchannel consisting of two parallel plates as shown in Fig.1. Assuming that the equilibrium Boltzmann distribution is applicable, it implies uniform dielectric constant and neglecting fluctuation. According to the theory of electrostatics, the relationship between the electrostatic potential $\psi_{i}$ and the charge density $\rho_{e i}$ is described by the Poisson equation. At any point in the fluid, it is described as (Mala et al., 1997; Hunter, 1981)

$$
\frac{d^{2} \psi_{i}}{d y^{2}}=-\frac{\rho_{e i}}{\varepsilon_{0} \varepsilon_{i}}, \quad i=1,2
$$

where the subscripts $i=1,2$ denote the values for regions I and II, respectively

For the case of any fluid consisting of two kinds of ions of equal and opposite charge $z_{i}^{+}, z_{i}^{-}$, the number of ions of each type are given by the Boltzmann equation

$$
n_{i}^{-}=n_{0 i} \exp \left(\frac{\hat{z}_{i} e \psi_{i}}{k_{b} \hat{T}}\right) \text { and } n_{i}^{+}=n_{0 i} \exp \left(-\frac{\hat{z}_{i} e \psi_{i}}{k_{b} \hat{T}}\right), i=1,2 \text {, }
$$

where $n_{0 i}$ and $\hat{z}_{i}$ are the bulk ionic concentrations and the valence of type $i$ ions, respectively, $e$ denotes the charge of a proton, $\psi_{i}$ are the electrostatic potentials. $k_{b}$ and $\hat{T}$ are the Boltzmann constant and the absolute temperature, respectively. Then the net charge density $\rho_{e i}$ in a unit volume of the fluid in both regions can be written as

$$
\rho_{e i}=\left(n_{i}^{+}-n_{i}^{-}\right) \hat{z}_{i} e=-2 n_{0 i} \hat{z}_{i} e \operatorname{Sinh}\left(\frac{\hat{z}_{i} e \psi_{i}}{k_{b} \hat{T}}\right), \quad i=1,2 .
$$


Substituting Eq.(20) into the Poisson equation (18), the well-known PoissonBoltzmann equation is obtained

$$
\frac{d^{2} \psi_{i}}{d y^{2}}=\frac{2 n_{0 i} \hat{z}_{i} e}{\varepsilon_{0} \varepsilon_{i}} \operatorname{Sinh}\left(\frac{\hat{z}_{i} e \psi_{i}}{k_{b} \hat{T}}\right), \quad i=1,2 .
$$

Introducing a set of dimensionless quantities as follows

$$
Y=\frac{y}{h_{1}}, \quad \Psi_{i}(Y)=\frac{\hat{z}_{i} e \psi_{i}}{k_{b} \hat{T}}, \quad \rho_{e i}^{*}(Y)=\frac{\rho_{e i}}{n_{0 i} \hat{z}_{i} e} .
$$

and through nondimensionalization, Eq.(21) can be non-dimensionalized to the following forms $\frac{d^{2} \Psi_{1}(Y)}{d Y^{2}}=\kappa_{1}^{2} \operatorname{Sinh}\left(\Psi_{1}(Y)\right), \frac{d^{2} \Psi_{1}(Y)}{d Y^{2}}=-\frac{\kappa_{1}^{2}}{2} \rho_{e 1}^{*}(Y), \quad 0<Y<1$.

$$
\frac{d^{2} \Psi_{2}(Y)}{d Y^{2}}=h^{2} \kappa_{2}^{2} \operatorname{Sinh}\left(\Psi_{2}(Y)\right), \frac{d^{2} \Psi_{2}(Y)}{d Y^{2}}=-\frac{h^{2} \kappa_{2}^{2}}{2} \rho_{e 2}^{*}(Y),-\frac{1}{h}<Y<0 .
$$

where $h=h_{1} / h_{2}, \kappa_{i}=h_{i} k_{i}$, in which $k_{i}=\left(2 n_{0 i} \hat{z}_{i}^{2} e^{2} / \varepsilon_{0} \varepsilon_{i} k_{b} \hat{T}\right)^{1 / 2}$ is the Debye-Hückel parameter, and $1 / k_{i}$ is generally regarded as the EDL thickness $(i=1,2)$.

According to the Debye-Hückel linear approximation, the electric potential is typically much smaller than the thermal energy of the ions, i.e. $\left|\hat{z}_{i} e \psi_{i}\right| \ll\left|k_{b} \hat{T}\right|$, such that the hyperbolic sine function in Eqs.(23) and (24) can be approximated by the first term in a Taylor series. Thus, Eqs.(23) and (24) can be rewritten as

$$
\begin{gathered}
\frac{d^{2} \Psi_{1}(Y)}{d Y^{2}}=\kappa_{1}^{2} \Psi_{1}(Y), \quad 0<Y<1, \\
\frac{d^{2} \Psi_{2}(Y)}{d Y^{2}}=h^{2} \kappa_{2}^{2} \Psi_{2}(Y), \quad-\frac{1}{h}<Y<0,
\end{gathered}
$$

The non-dimensional boundary conditions of Eqs.(25) and (26) can be obtained from Eq.(17), as

$$
\begin{gathered}
\Psi_{1}(1)=\frac{\hat{z}_{1} e \zeta_{1}}{k_{b} \hat{T}}, \quad \Psi_{2}\left(-\frac{1}{h}\right)=\frac{\hat{z}_{2} e \zeta_{2}}{k_{b} \hat{T}}, \\
\Psi_{1}(0)=\hat{z} \Psi_{2}(0),\left.\quad \frac{d \Psi_{1}(Y)}{d Y}\right|_{Y=0}=\left.\frac{\hat{z}}{\varepsilon} \frac{d \Psi_{2}(Y)}{d Y}\right|_{Y=0},
\end{gathered}
$$

where $\hat{z}=\hat{z}_{1} / \hat{z}_{2}, \varepsilon=\varepsilon_{1} / \varepsilon_{2}$.

With the above boundary conditions (27) and (28), the solutions of Eqs.(25) and (26) are obtained 


$$
\begin{aligned}
\Psi_{1}(Y)= & {\left[\varepsilon \kappa_{1} \bar{\zeta}_{1} \operatorname{Sinh} \kappa_{2} \operatorname{Cosh}\left(\kappa_{1} Y\right)+h \kappa_{2} \bar{\zeta}_{1} \operatorname{Cosh} \kappa_{2} \operatorname{Sinh}\left(\kappa_{1} Y\right),\right.} \\
& \left.+h \kappa_{2} \hat{z} \bar{\zeta}_{2} \operatorname{Sinh}\left(\kappa_{1}-\kappa_{1} Y\right)\right] / C_{\kappa} \\
\Psi_{2}(Y)= & {\left[\varepsilon \kappa_{1} \bar{\zeta}_{1} \operatorname{Sinh}\left(\kappa_{2}+h \kappa_{2} Y\right)-\varepsilon \kappa_{1} \hat{z} \bar{\zeta} \operatorname{Cosh} \kappa_{1} \operatorname{Sinh}\left(h \kappa_{2} Y\right)\right.} \\
& \left.+h \kappa_{2} \hat{z} \bar{\zeta}_{2} \operatorname{Sinh} \kappa_{1} \operatorname{Cosh}\left(h \kappa_{2} Y\right)\right] /\left(\hat{z} C_{\kappa}\right)
\end{aligned}
$$

where $\quad C_{\kappa}=\varepsilon \kappa_{1} \operatorname{Sinh} \kappa_{2} \operatorname{Cosh} \kappa_{1}+h \kappa_{2} \operatorname{Cosh} \kappa_{2} \operatorname{Sinh} \kappa_{1} \quad$ and $\quad \bar{\zeta}_{1}=\hat{z}_{1} e \zeta_{1} /\left(k_{b} \hat{T}\right)$, $\bar{\zeta}_{2}=\hat{z}_{2} e \zeta_{2} / k_{b} \hat{T}$ are constants.

\subsection{The dimensionless form of momentum, thermal energy and nanoparticles equations}

For the convenience of mathematical calculation and in-depth analysis of the physical mechanism, it is necessary to nondimensionalize the governing equations. Define the non-dimensional quantities as follows $(i=1,2)$

$$
Y=\frac{y}{h_{1}}, U_{i}(Y)=\frac{u_{i}}{u_{m}}, \theta_{i}(Y)=\frac{T_{i}-T_{0}}{T_{w i}-T_{0}}, \phi(Y)=\frac{C-C_{0}}{C_{1}-C_{0}},
$$

where, $u_{m}$ is the average velocity at the entrance, $C_{0}$ is the reference nanoparticle volume fraction.

Non-dimensionalize the momentum Eqs.(11) and (14) by the non-dimensional quantities (22) and (31), we obtain

$$
\begin{gathered}
\frac{d^{2} U_{1}}{d Y^{2}}+P_{1}-2 G_{1} \bar{E}_{s 1} \Psi_{1}-H a_{1}^{2} U_{1}=0 \\
\frac{d^{2} U_{2}}{d Y^{2}}+h^{2} P_{2}-2 h^{2} G_{2} \bar{E}_{s 2} \Psi_{2}-h^{2} H a_{2}^{2} U_{2}=0
\end{gathered}
$$

subject to the boundary conditions,

$$
U_{1}(1)=0, \quad U_{1}(0)=U_{2}(0),\left.\quad \frac{d U_{1}(Y)}{d Y}\right|_{Y=0}=\left.\frac{1}{\mu} \frac{d U_{2}(Y)}{d Y}\right|_{Y=0}, U_{2}\left(-\frac{1}{h}\right)=0,
$$

where, $h=h_{1} / h_{2}, \mu=\mu_{1} / \mu_{2}, \quad G_{1}$ and $G_{2}$ are constants, $H a_{i}=B_{0} h_{i} \sqrt{\sigma_{i} / \mu_{i}}$ $(i=1,2)$ are the non-dimensional Hartmann numbers in regions I and II, respectively, which is a measure of the strength of the applied magnetic field. The parameters $P_{i}$ and $\bar{E}_{s i} \quad(i=1,2)$ are the non-dimensional pressure gradient parameters and the streaming potentials in regions I and II, respectively, and are defined by 


$$
P_{i}=-\frac{h_{i}^{2}}{\mu_{i} u_{m}} \frac{\partial p_{i}}{\partial x_{i}}, \quad \bar{E}_{s i}=\frac{E_{s i}}{\zeta_{i}}=\frac{E_{x i} L}{\zeta_{i}}, G_{1}=\frac{n_{01} \hat{z}_{1} e \zeta_{1} h_{1}^{2}}{\mu_{1} u_{m} L}, G_{2}=\frac{n_{02} \hat{z}_{2} e \zeta_{2} h_{2}^{2}}{\mu_{2} u_{m} L}
$$

As seen in Eqs.(32) and (33), the streaming potential $\bar{E}_{s 1}$ and $\bar{E}_{s 2}$ are unknown. It is noted that as the electrolyte flows over a solid surface, free charged particles in the EDL surface will move along the direction of liquid flow. The movement of these charged particles leads to the accumulation of charges downstream. Thus, a potential difference is formed between the upstream and downstream. This potential is known as streaming potential. Correspondingly, the current produced by the movement of charged particles is called streaming current, defined by

$$
\begin{aligned}
& I_{s 1}=\int_{\Omega_{1}} u_{1} \rho_{e 1} d \Omega_{1}, \quad \text { in Region I } \\
& I_{s 2}=\int_{\Omega_{2}} u_{2} \rho_{e 2} d \Omega_{2}, \quad \text { in Region II }
\end{aligned}
$$

here, $\Omega_{1}$ and $\Omega_{2}$ are the cross-sectional areas of the regions I and II, and $\Omega_{i}=h_{i} W$ $(i=1,2), W$ is the width of the microchannel.

Using Eqs.(22)-(24) and (31), the dimensionless form of streaming current is obtained as

$$
\begin{gathered}
I_{s 1}=-2 W h_{1} u_{m} n_{01} \hat{z}_{1} e \int_{0}^{1} U_{1}(Y) \Psi_{1}(Y) d Y, \\
I_{s 2}=-2 W h_{1} u_{m} n_{02} \hat{z}_{2} e \int_{-1 / h}^{0} U_{2}(Y) \Psi_{2}(Y) d Y,
\end{gathered}
$$

Considering that the streaming potential generated by the streaming current will produce a conduction current in the reverse direction, thus

$$
\begin{array}{ll}
I_{c 1}=\frac{\lambda_{01} E_{s 1} \Omega_{1}}{L}, & \text { in Region I } \\
I_{c 2}=\frac{\lambda_{02} E_{s 2} \Omega_{2}}{L}, & \text { in Region II }
\end{array}
$$

where, $\lambda_{01}$ and $\lambda_{02}$ are the electrical conductivities of the fluid in regions I and II.

Using the relations $\bar{E}_{s i}=E_{s i} / \zeta_{i}$ and $\Omega_{i}=h_{i} W(i=1,2)$, the non-dimensional conduction current is given by

$$
I_{c 1}=\frac{\lambda_{01} \zeta_{1} \bar{E}_{s 1} h_{1} W}{L}
$$




$$
I_{c 2}=\frac{\lambda_{02} \zeta_{2} \bar{E}_{s 2} h_{2} W}{L}
$$

Usually the net electrical current is the algebraic summation of the streaming current and the conduction current. In a steady state, this net electrical current should be zero, which indicates

$$
\begin{gathered}
I_{s 1}+I_{c 1}=0, \quad \text { in Region I } \\
I_{s 2}+I_{c 2}=0, \quad \text { in Region II }
\end{gathered}
$$

Therefore, the electrokinetic potential $E_{x i}(i=1,2)$ can be obtained by balancing the streaming current and conduction current at the steady state. Using Eqs.(37)-(38) and Eqs.(41)-(42), $\bar{E}_{s 1}$ and $\bar{E}_{s 2}$ are obtained

$$
\begin{array}{cc}
\bar{E}_{s 1}=2 G_{3} \int_{0}^{1} U_{1}(Y) \Psi_{1}(Y) d Y, & \text { in Region I } \\
\bar{E}_{s 2}=2 h G_{4} \int_{-1 / h}^{0} U_{2}(Y) \Psi_{2}(Y) d Y, & \text { in Region II }
\end{array}
$$

where $G_{3}=u_{m} n_{01} \hat{z}_{1} e L /\left(\lambda_{01} \zeta_{1}\right)$ and $G_{4}=u_{m} n_{02} \hat{z}_{2} e L /\left(\lambda_{02} \zeta_{2}\right)$ are constants.

Therefore, Eqs.(32)-(33) are transformed into the following form

$$
\begin{gathered}
\frac{d^{2} U_{1}}{d Y^{2}}+P_{1}-4 G_{1} G_{3} \int_{0}^{1} U_{1} \Psi_{1} d Y \Psi_{1}-H a_{1}^{2} U_{1}=0, \quad 0<Y<1 . \\
\frac{d^{2} U_{2}}{d Y^{2}}+h^{2} P_{2}-4 h^{3} G_{2} G_{4} \int_{-1 / h}^{0} U_{2} \Psi_{2} d Y \Psi_{2}-h^{2} H a_{2}^{2} U_{2}=0, \quad-\frac{1}{h}<Y<0 .
\end{gathered}
$$

Similarly, substituting the non-dimensional variables (31) into Eqs.(12) and (15)(16), we obtained the reduced energy equations and concentration of nanoparticles equation as

$$
\begin{gathered}
\frac{d^{2} \theta_{1}}{d Y^{2}}+B r_{1}\left(\frac{d U_{1}}{d Y}\right)^{2}=0, \quad 0<Y<1, \\
\frac{d^{2} \theta_{2}}{d Y^{2}}+N b \frac{d \theta_{2}}{d Y} \frac{d \phi}{d Y}+N t\left(\frac{d \theta_{2}}{d Y}\right)^{2}+B r_{2}\left(\frac{d U_{2}}{d Y}\right)^{2}=0, \quad-\frac{1}{h}<Y<0, \\
\frac{d^{2} \phi}{d Y^{2}}+\frac{N t}{N b} \frac{d^{2} \theta_{2}}{d Y^{2}}=0, \quad-\frac{1}{h}<Y<0,
\end{gathered}
$$

The corresponding boundary conditions in non-dimensional form are 


$$
\begin{aligned}
& \theta_{1}(1)=1, \quad \theta_{1}(0)=\delta \theta_{2}(0), \quad \theta_{2}(-1 / h)=1, \quad \phi(0)=1, \\
& \left.\frac{d \theta_{1}}{d Y}\right|_{Y=0}=\left.\frac{\delta d \theta_{2}}{k_{f} d Y}\right|_{Y=0},\left.N b \frac{d \phi}{d Y}\right|_{Y=-\frac{1}{h}}+\left.N t \frac{d \theta_{2}}{d Y}\right|_{Y=-\frac{1}{h}}=0,
\end{aligned}
$$

where, $\delta=\left(T_{w 2}-T_{0}\right) /\left(T_{w 1}-T_{0}\right)$ and $k_{f}=k_{f 1} / k_{f 2}$ are constants, the parameters $\mathrm{Br}_{i}(i=1,2), N b$ and $N t$ are the Brinkman number, the Brownian motion parameter and the thermophoresis parameter, respectively, and they are defined by

$$
B r_{i}=P r_{i} \cdot E c_{i}, N b=\frac{\tau D_{B}\left(C_{1}-C_{0}\right)}{\alpha_{2}}, N t=\frac{\tau D_{T}\left(T_{w 2}-T_{0}\right)}{\alpha_{2} T_{0}},
$$

in which $\operatorname{Pr}_{i}=v_{i} / \alpha_{i}$ and $E c_{i}=u_{m}^{2} /\left[c_{p i}\left(T_{w i}-T_{0}\right)\right],(i=1,2)$ are the Prandtl number and Eckert number in regions I and II, respectively.

\subsection{Important physical quantities}

It is noted that any real fluids flowing along a solid boundary will incur a shear stress at the boundary. Besides, it is necessary to understand convective heat transfer between the microchannel surface and the fluid flowing past it. Therefore, the physically important quantities of practical interests are the local skin friction, the local Nusselt number and the local Sherwood number. In present work, they are defined as follows $(i=1,2)$

$$
C_{f i}=\frac{\tau_{w i}}{\frac{1}{2} \rho_{i} u_{m}^{2}}, \quad N u_{i}=\frac{h_{i} q_{w i}}{k_{f i}\left(T_{w i}-T_{0}\right)}, \quad S h=\frac{h_{2} q_{C}}{D_{B}\left(C_{1}-C_{0}\right)},
$$

where,

$$
\tau_{w i}=\left.\mu_{i} \frac{d u_{i}}{d y}\right|_{y=(-1)^{1+i} h_{i}}, \quad q_{w i}=-\left.k_{f i} \frac{d T_{i}}{d y}\right|_{y=(-1)^{1+i} h_{i}}, \quad q_{C}=-\left.D_{B} \frac{d C}{d y}\right|_{y=-h_{2}} .
$$

Substituting Eqs.(31) and (55) into Eq. (54), we obtain

$$
\begin{aligned}
& \operatorname{Re}_{1} C_{f 1}=2 U_{1}^{\prime}(1), \quad \operatorname{Re}_{2} C_{f 2}=\frac{2}{h} U_{2}^{\prime}\left(-\frac{1}{h}\right), \quad N u_{1}=-\theta_{1}^{\prime}(1), \\
& N u_{2}=-\frac{1}{h} \theta_{2}^{\prime}\left(-\frac{1}{h}\right), \quad S h=-\frac{1}{h} \phi^{\prime}\left(-\frac{1}{h}\right)
\end{aligned}
$$

where $\operatorname{Re}_{i}=\rho_{i} u_{m} h_{i} / \mu_{i} \quad(i=1,2)$ are the Reynolds numbers in regions I and II, respectively. 


\section{Results and discussion}

The homotopy analysis method(HAM) is applied to obtain solutions to the problem defined in the previous section. It is worth mentioning that Eqs.(47) and (48) contain two unknown constants $\int_{0}^{1} U_{1} \Psi_{1} d Y$ and $\int_{-1 / h}^{0} U_{2} \Psi_{2} d Y$. The integral terms are typically difficult to calculate directly by either numerical or analytical methods. To overcome this difficulty, in the present approach, extension has been made to the HAM to obtain the accurate solutions of the nonlinear equations (25)-(28) and (47)-(52). In the present computational process, the exact values for $\int_{0}^{1} U_{1} \Psi_{1} d Y$ and $\int_{-1 / h}^{0} U_{2} \Psi_{2} d Y$ are obtained spontaneously without any approximations. Unlike the other analytic techniques, the HAM provides us a convenient way to control and adjust the convergence of solution series, so that the convergence of solution series can be guaranteed. The details are shown in the Appendix.

To verify the accuracy of the results, the maximum total average squared residual error function is defined as

$$
E(m)=\max \left\{E_{\Psi_{i}}(m), E_{U_{i}}(m), E_{\theta_{i}}(m), E_{\phi}(m)\right\}, \quad i=1,2
$$

where

$$
\begin{aligned}
& E_{\Psi_{1}}(m)=\int_{0}^{1}\left(\Psi_{1}^{\prime \prime}-\kappa_{1}^{2} \Psi_{1}\right)^{2} d Y, \quad E_{\Psi_{2}}(m)=\int_{-1 / h}^{0}\left(\Psi_{2}^{\prime \prime}-h^{2} \kappa_{2}^{2} \Psi_{2}\right)^{2} d Y, \\
& E_{U_{1}}(m)=\int_{0}^{1}\left(U_{1}^{\prime \prime}+P_{1}-4 G_{1} G_{3} \int_{0}^{1} U_{1} \Psi_{1} d Y \Psi_{1}-H a_{1}^{2} U_{1}\right)^{2} d Y, \\
& E_{U_{2}}(m)=\int_{-1 / h}^{0}\left(U_{2}^{\prime \prime}+h^{2} P_{2}-4 h^{3} G_{2} G_{4} \int_{-1 / h}^{0} U_{2} \Psi_{2} d Y \Psi_{2}-h^{2} H a_{2}^{2} U_{2}\right)^{2} d Y, \\
& E_{\theta_{1}}(m)=\int_{0}^{1}\left(\theta_{1}^{\prime \prime}+B r_{1} U_{1}^{\prime 2}\right)^{2} d Y \\
& E_{\theta_{2}}(m)=\int_{-1 / h}^{0}\left(\theta_{2}^{\prime \prime}+N b \theta_{2}^{\prime} \phi^{\prime}+N t \theta_{2}^{\prime 2}+B r_{2} U_{2}^{\prime 2}\right)^{2} d Y, \\
& E_{\phi}(m)=\int_{-1 / h}^{0}\left(\phi^{\prime \prime}+\frac{N t}{N b} \theta_{2}^{\prime \prime}\right)^{2} d Y
\end{aligned}
$$

in which, $m$ is the computational order.

Substituting $m$ th order solutions into Eq.(57), the corresponding error can be obtained. In order to facilitate calculation, the constants and the dimensionless parameters are given the fixed values unless otherwise specified in the present work including $G_{1}=G_{2}=G_{3}=G_{4}=1, \bar{\zeta}_{1}=\bar{\zeta}_{2}=1, \mu=k_{f}=\varepsilon=\hat{z}=\delta=1, P_{1}=P_{2}=1$. It 
is worth noting that the distance ratio of the two-layer fluid $h$ was commonly chosen as 1 in most previous studies (Shit et al., 2016; Xie and Jian, 2017) implying that the two-layer fluid have the same spatial scales. However, in a real engineering case, the space scale occupied by the two layers of liquid are often different. In view of this, the different spatial scales for the two-layer fluid are considered in the present study, namely, $h=2$. In this case, we obtain the maximum error $E(m)$ for various values of $\kappa_{1}$ and $\kappa_{2}$, as listed in Table I and Table II. It is shown that the maximum error continues to decline with the increase of calculating order demonstrating that the accuracy of the results is guaranteed. Therefore, the 40th order calculation results were adopted for analysis and discussion in present work. It is noted that when the value of $\kappa_{1}$ or $\kappa_{2}$ is greater than 5 , the error convergence of the potential equation tends to be slow, which subsequently leads to the slow convergence of other equations. In these cases, the Homotopy-Padé technique (Liao, 2012) is employed to accelerate the convergence of the HAM approximations.

Table I. The maximum error $E(m)$ for different $\kappa_{1}$ in the case of $\kappa_{2}=1, H a_{1}=H a_{2}=1$,

\begin{tabular}{|c|c|c|c|c|c|}
\multicolumn{7}{|c|}{$B r_{1}=B r_{2}=1$ and $N b=N t=0.1$} \\
\hline$E(m)$ & \multicolumn{5}{|c|}{$\kappa_{2}=1$} \\
\hline Order & $\kappa_{1}=1$ & $\kappa_{1}=2$ & $\kappa_{1}=3$ & $\kappa_{1}=5$ & $\kappa_{1}=10$ \\
\hline$m=10$ & 0.0391 & 0.0049 & 0.0064 & 0.3116 & 113.111 \\
\hline$m=20$ & $1.736 \times 10^{-5}$ & $8.908 \times 10^{-9}$ & $1.205 \times 10^{-8}$ & $4.050 \times 10^{-7}$ & $2.250 \times 10^{-5}$ \\
\hline$m=30$ & $6.248 \times 10^{-9}$ & $1.358 \times 10^{-13}$ & $7.908 \times 10^{-13}$ & $6.312 \times 10^{-10}$ & $3.683 \times 10^{-7}$ \\
\hline$m=40$ & $6.351 \times 10^{-12}$ & $4.957 \times 10^{-18}$ & $3.267 \times 10^{-17}$ & $1.919 \times 10^{-12}$ & $7.055 \times 10^{-8}$ \\
\hline
\end{tabular}

Table II. The maximum error $E(m)$ for different $\kappa_{2}$ in the case of $\kappa_{1}=1, H a_{1}=H a_{2}=1$,

$$
B r_{1}=B r_{2}=1 \text { and } N b=N t=0.1 \text {. }
$$

\begin{tabular}{|c|c|c|c|c|c|}
\hline$E(m)$ & \multicolumn{5}{|c|}{$\kappa_{1}=1$} \\
\hline Order & $\kappa_{2}=1$ & $\kappa_{2}=2$ & $\kappa_{2}=3$ & $\kappa_{2}=5$ & $\kappa_{2}=10$ \\
\hline$m=10$ & 0.0391 & $3.671 \times 10^{-4}$ & 0.0044 & 0.0079 & 531.073 \\
\hline$m=20$ & $1.736 \times 10^{-5}$ & $1.966 \times 10^{-8}$ & $1.469 \times 10^{-6}$ & $4.438 \times 10^{-7}$ & $8.150 \times 10^{-7}$ \\
\hline$m=30$ & $6.248 \times 10^{-9}$ & $4.219 \times 10^{-13}$ & $3.612 \times 10^{-10}$ & $3.521 \times 10^{-9}$ & $2.561 \times 10^{-7}$ \\
\hline$m=40$ & $6.351 \times 10^{-12}$ & $1.570 \times 10^{-17}$ & $9.436 \times 10^{-14}$ & $2.516 \times 10^{-11}$ & $1.630 \times 10^{-8}$ \\
\hline
\end{tabular}




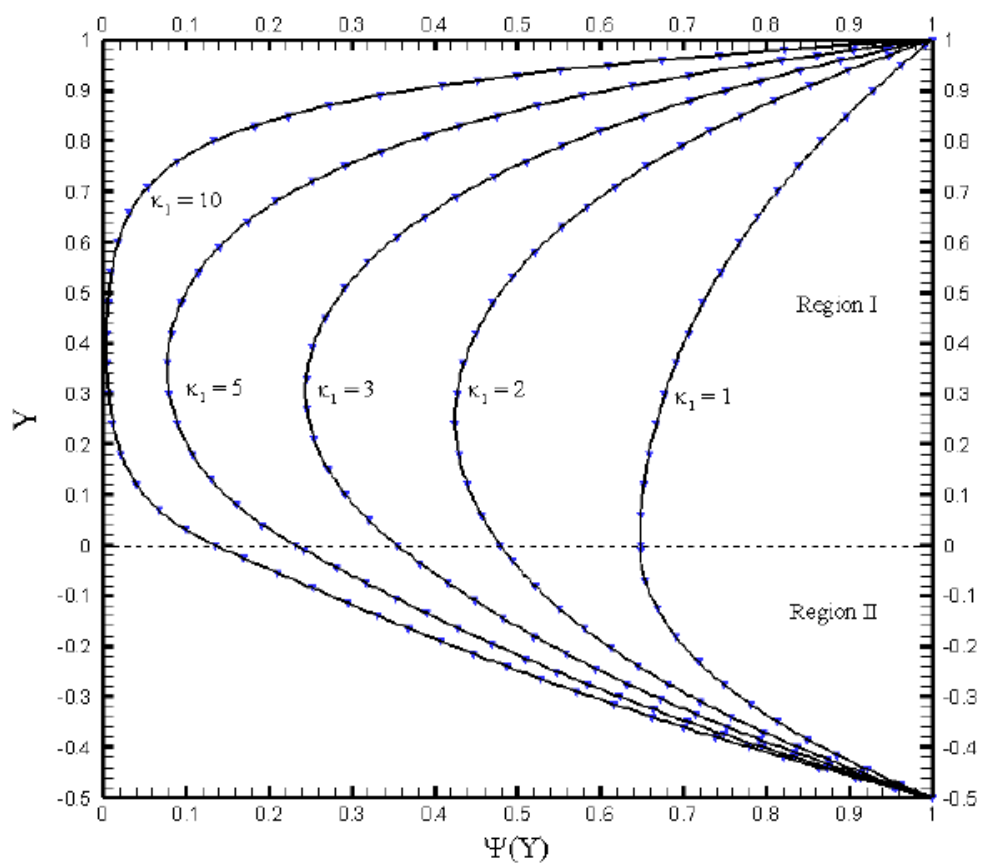

(a) For various values of $\kappa_{1}$ with $\kappa_{2}=1$

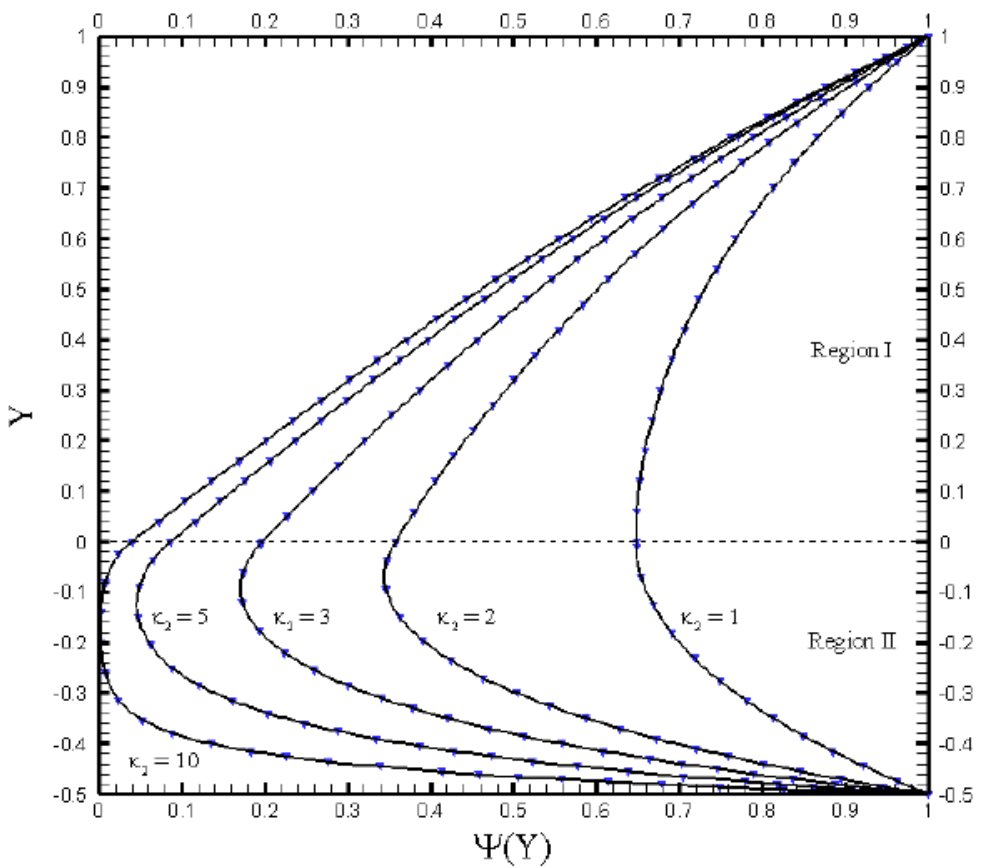

(b) For various values of $\kappa_{2}$ with $\kappa_{1}=1$

Fig.2 The electrical potential $\Psi(Y)$ with $\varepsilon=\hat{z}=1, \bar{\zeta}_{1}=\bar{\zeta}_{2}=1, h=2$. Line: analytical solutions given by Eqs.(29) and (30); Triangle symbols: HAM solutions.

In Fig.2(a) and Fig.2(b), the electrical potential profiles are presented for different values of the parameter $\kappa_{1}$ and $\kappa_{2}$ respectively. It is noticed that the present HAM 
solutions agree with the analytical ones given by (29) and (30) in the whole region $-0.5 \leq Y \leq 1$ for all considered values of $\kappa_{1}$ and $\kappa_{2}$. This further confirms the validity and accuracy of the present solution procedures. As shown in Fig. 2, the solid lines represent exact solutions which given by (29) and (30) and the symbols are the results of the present HAM approximation. It can be observed that the electrical potential profiles $\Psi(Y)$ are considerably reduced with increasing $\kappa_{1}$ and $\kappa_{2}$. As seen in Fig.2(a), as $\kappa_{1}$ becomes sufficiently large, $\Psi(Y)$ diminishes to zero near the middle of the channel. The reason causing this phenomenon is that the larger is $\kappa_{1}$, the thinner is the EDL. As $\kappa_{1}$ is considerably large, the thickness of EDL approaches to zero on the upper wall of the microchannel. Therefore, the electrical potential near the upper wall of the microchannel will decreases rapidly with $\kappa_{1}$ increasing. Similar phenomena can also be observed in Fig.2(b).

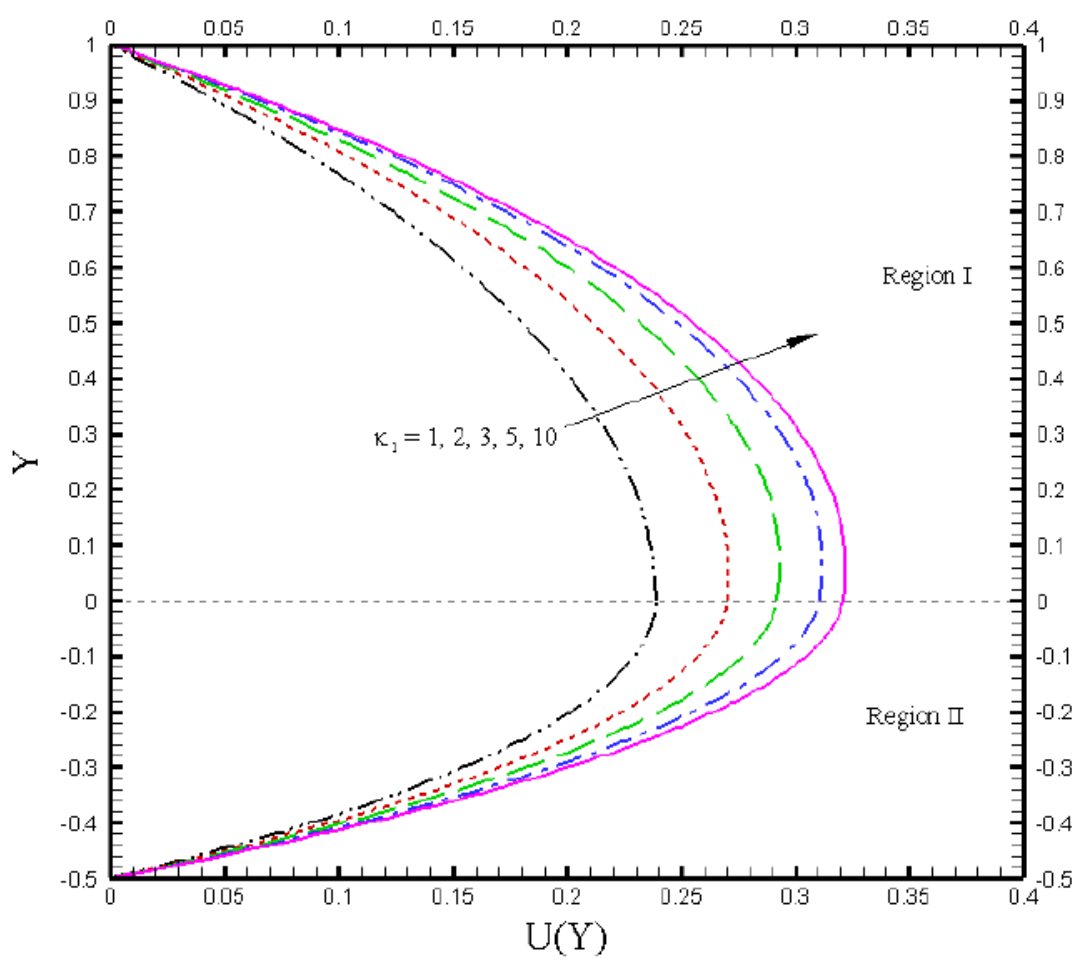

(a) For various values of $\kappa_{1}$ with $\kappa_{2}=1$ 


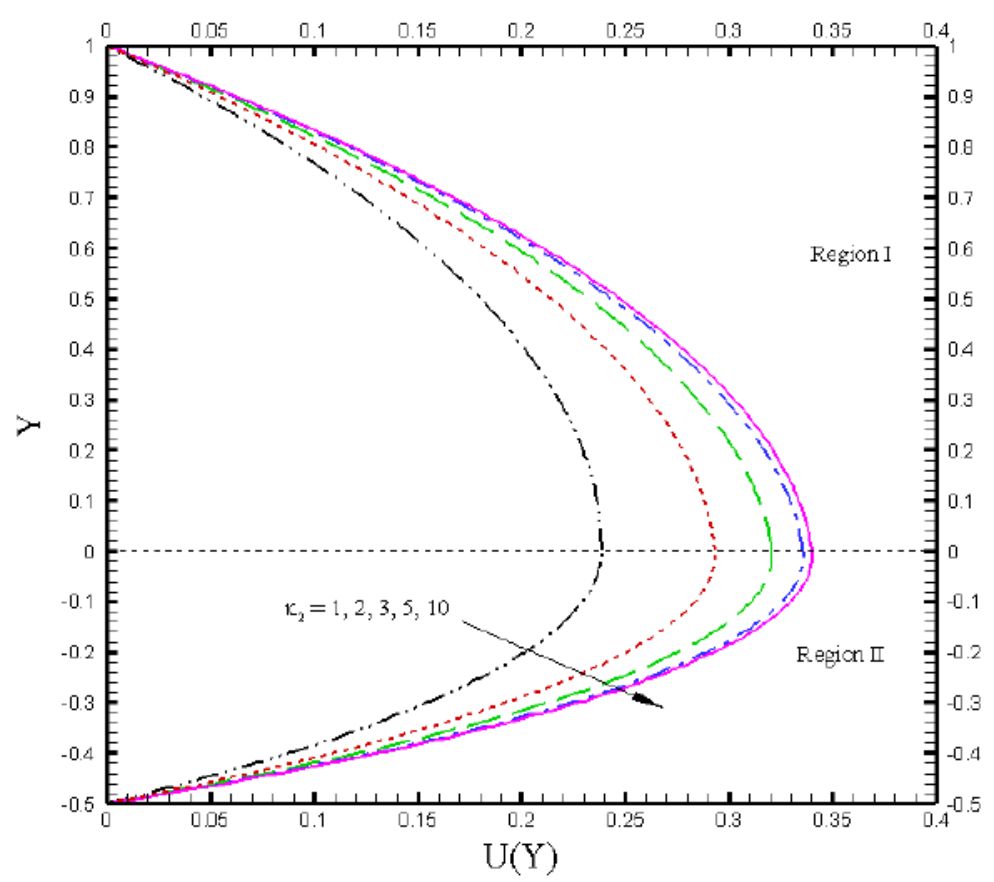

(b) For various values of $\kappa_{2}$ with $\kappa_{1}=1$

Fig.3 The dimensionless velocity profiles $U(Y)$ in the case of $G_{1}=G_{2}=G_{3}=G_{4}=1$, $P_{1}=P_{2}=1, H a_{1}=H a_{2}=1$ and $h=2$.

Fig.3(a) and Fig.3(b) show the non-dimensional velocity distribution in the microchannel. The non-dimensional velocity is seen increasing with increasing $\kappa_{1}$ and $\kappa_{2}$. The reason for this is that increasing $\kappa_{1}$ or $\kappa_{2}$ implies either a large separation distance between the channel walls or a smaller EDL thickness resulting in larger portion of fluid not being affected by the EDL and indicates that increasing the EDL thickness apparently restrains the fluid flow. Therefore, the EDL modifies the velocity profile which will affect the heat transfer in the microchannel.

To investigate the relationship between EDL and heat transfer, the influence of $\kappa_{1}$ and $\kappa_{2}$ on the dimensionless temperature profiles and nanoparticle volume fraction profiles are presented in Fig.4(a) and Fig.4(b), respectively. In Fig.4(a), it is shown that $\theta(Y)$ increases gradually as $\kappa_{1}$ evolves. If the distance of the two-layer fluid remains unchanged, then $\kappa_{1}$ is inversely proportional to the thickness of EDL. In the case, the larger is $\kappa_{1}$, the thinner is the EDL. As seen from Fig.4(a), taking the value of $\kappa_{1}$ from 1 to 10 , the maximum value of $\theta(Y)$ varies from 1.0188 to 1.0341 . It indicates that the effect of EDL on the distribution of temperature is not significant. In other 
words, the effect of EDL on temperature is generally negligible. Similar trend can also be observed in Fig.4(b).

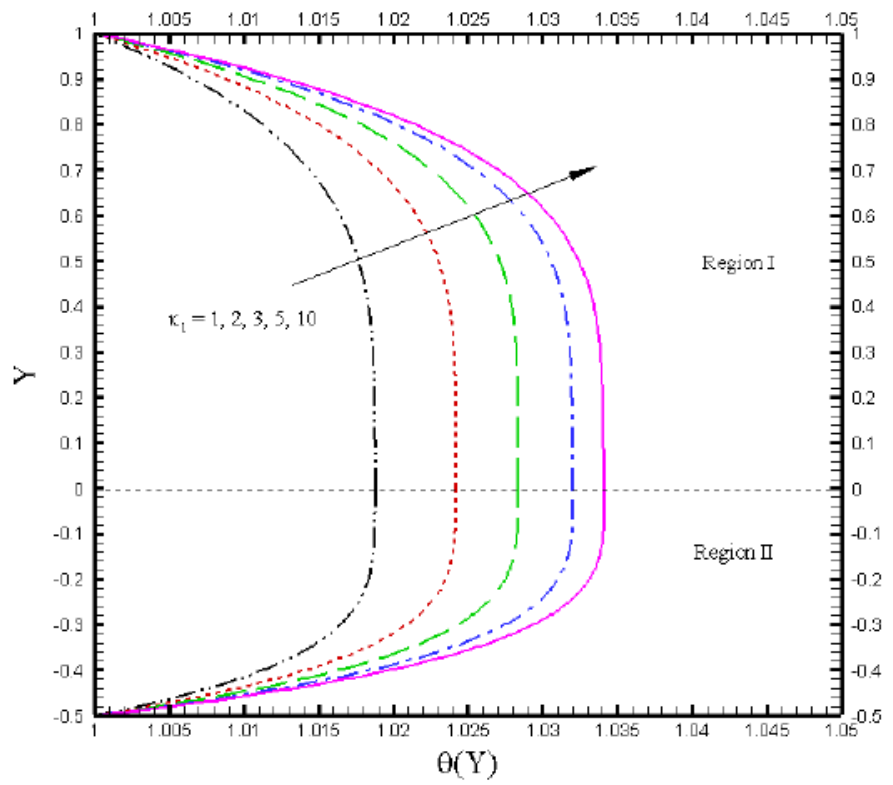

(a) For various values of $\kappa_{1}$ with $\kappa_{2}=1$

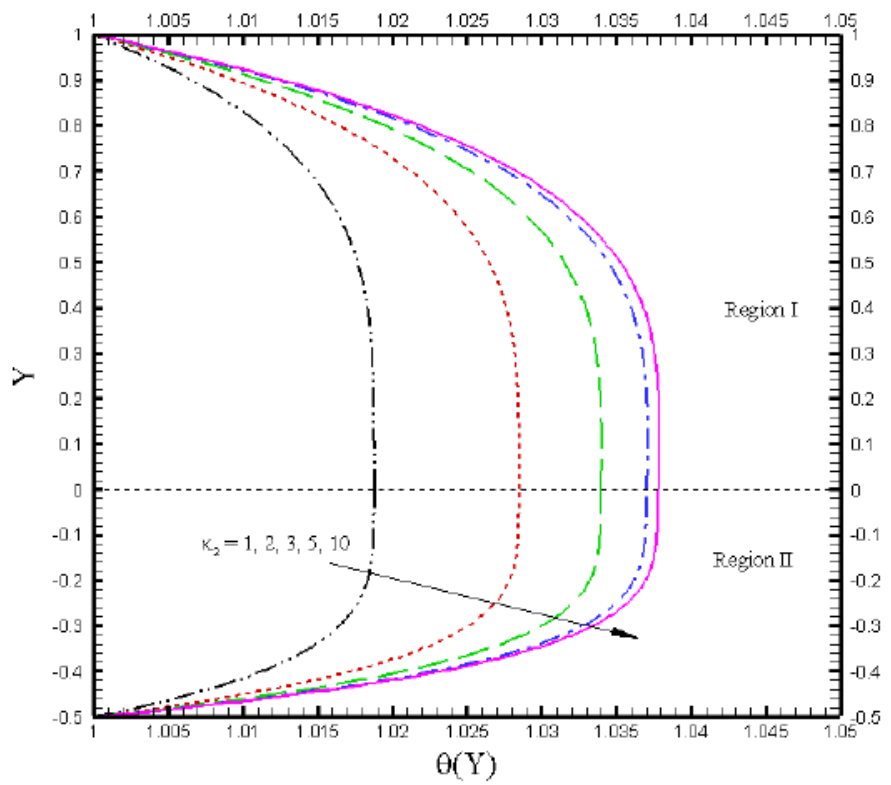

(b) For various values of $\kappa_{2}$ with $\kappa_{1}=1$

Fig.4 The dimensionless temperature profiles $\theta(Y)$ in the case of $H a_{1}=H a_{2}=1$, $N t=N b=0.1, B r_{1}=B r_{2}=1$ and $h=2$. 


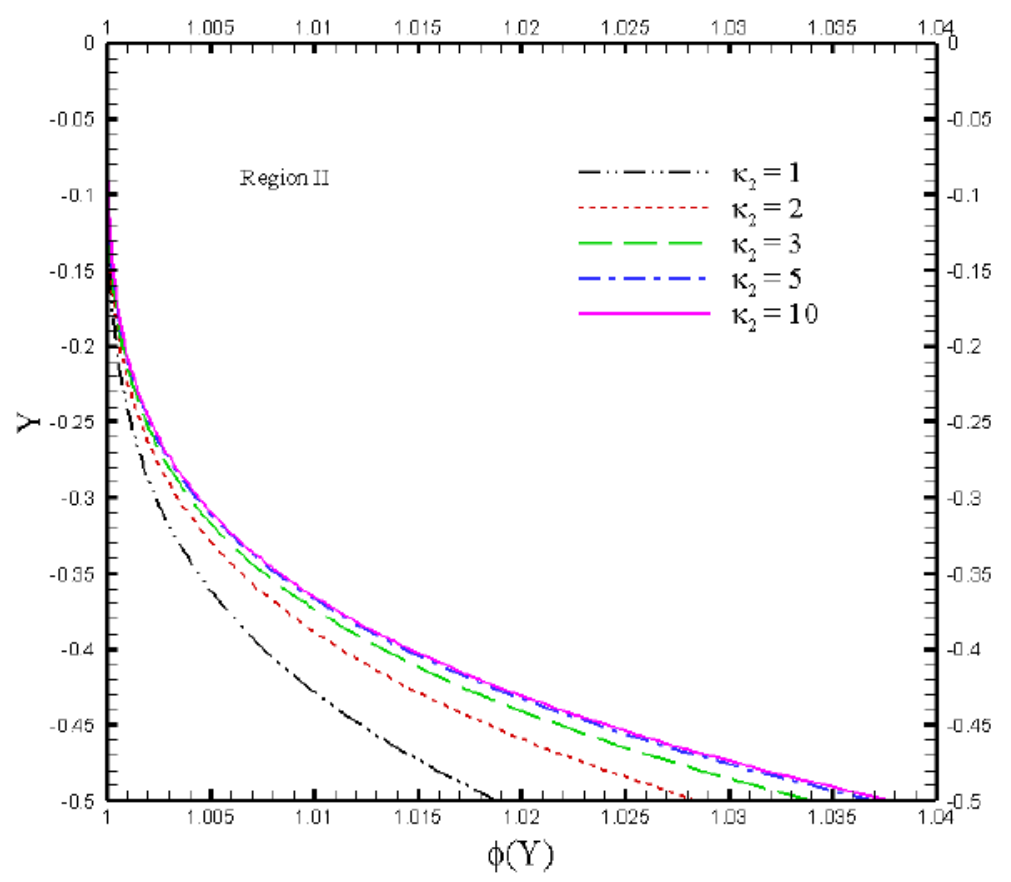

Fig.5 The nanoparticle volume fraction profiles $\phi(Y)$ for various values of $\kappa_{2}$ in the case of $\kappa_{1}=1, H a_{1}=H a_{2}=1, N t=N b=0.1, B r_{1}=B r_{2}=1$ and $h=2$.

Fig.5 illustrates the change in nanoparticle volume fraction profiles for various values of $\kappa_{2}$. It can be seen that the volume fraction of nanoparticles increases slightly with the increase of $\kappa_{2}$. As mentioned in the previous part, the larger is $\kappa_{2}$, the thinner is the EDL. It indicates that the EDL can influence the concentration of nanoparticles. Therefore, for some precision instruments, increasing the EDL effect can effectively reduce the attachment of nanoparticles to the wall. In addition, for sufficiently large parameter $\kappa_{2}$, the temperature distribution and nanoparticle concentration distribution curves will not change, as seen in Fig4(b) and Fig.5. It indicates that the EDL effect can be ignored when $\kappa_{2}$ is sufficiently large.

The influences of Hartmann number $\mathrm{Ha}_{1}$ and $\mathrm{Ha}_{2}$ on the distribution of dimensionless velocity are presented in Fig.6(a) and Fig.6(b). It is shown that the enlargement of the Hartmann number causes the reduction of the flow velocity in both regions of the microchannel. Since the Hartmann number is the square root of the ratio of electromagnetic force to the viscous force, the larger the Hartmann number is, the stronger the magnetic field strength will be. While the magnetic field force caused by fluid cutting magnetic field line and the viscous force are dominating in restraining the 
fluid flow. Consequently, the flow rate of the fluid is reduced with an increase in Hartmann number.

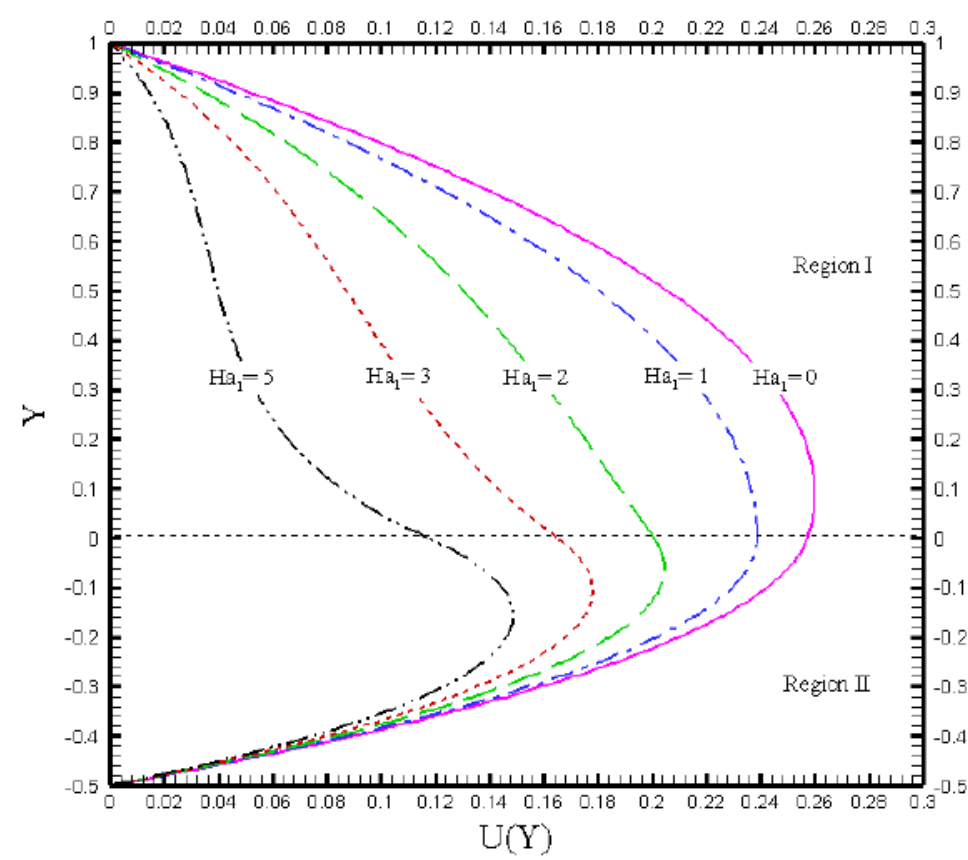

(a) For various values of $\mathrm{Ha}_{1}$ with $\mathrm{Ha}_{2}=1$

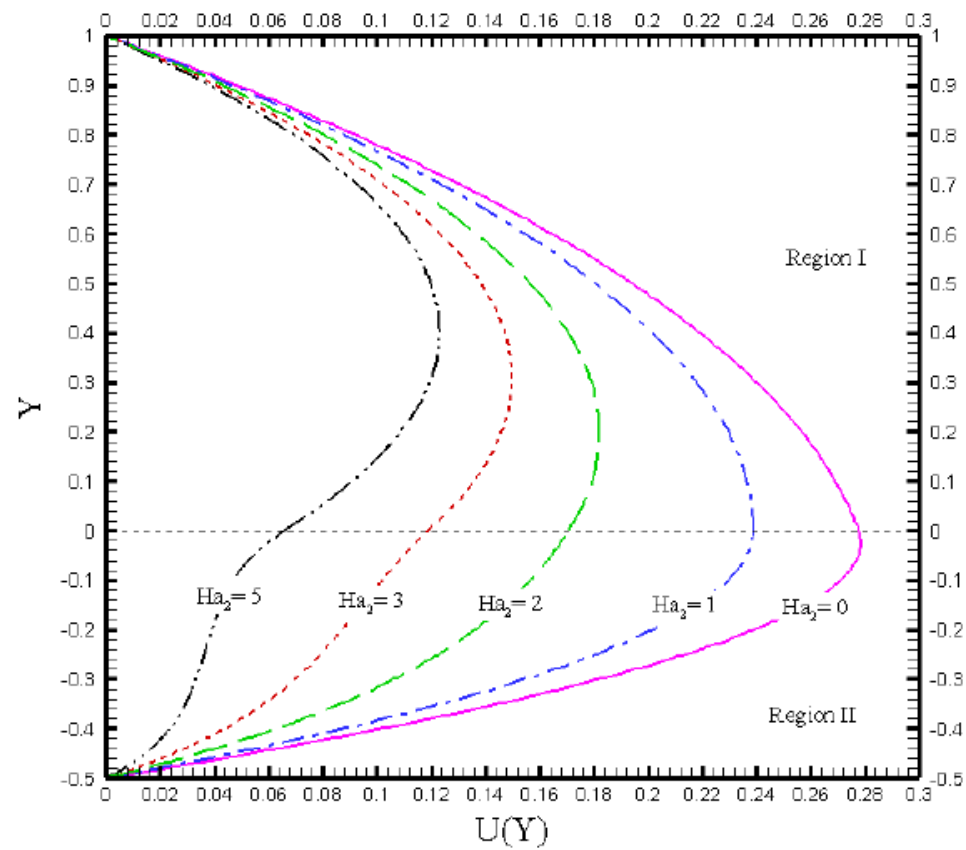

(b) For various values of $H a_{2}$ with $H a_{1}=1$

Fig.6 The dimensionless velocity profiles $U(Y)$ in the case of $\kappa_{1}=\kappa_{2}=1$ and $h=2$. 


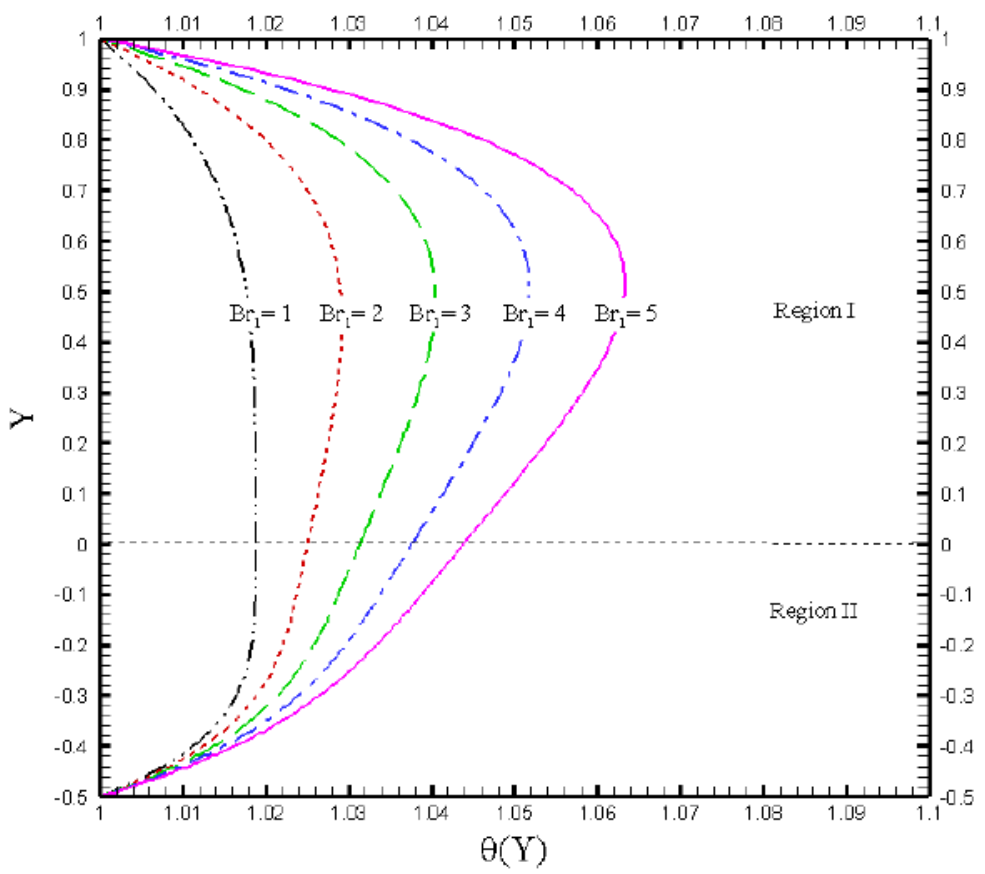

(a) For various values of $B r_{1}$ with $B r_{2}=1$

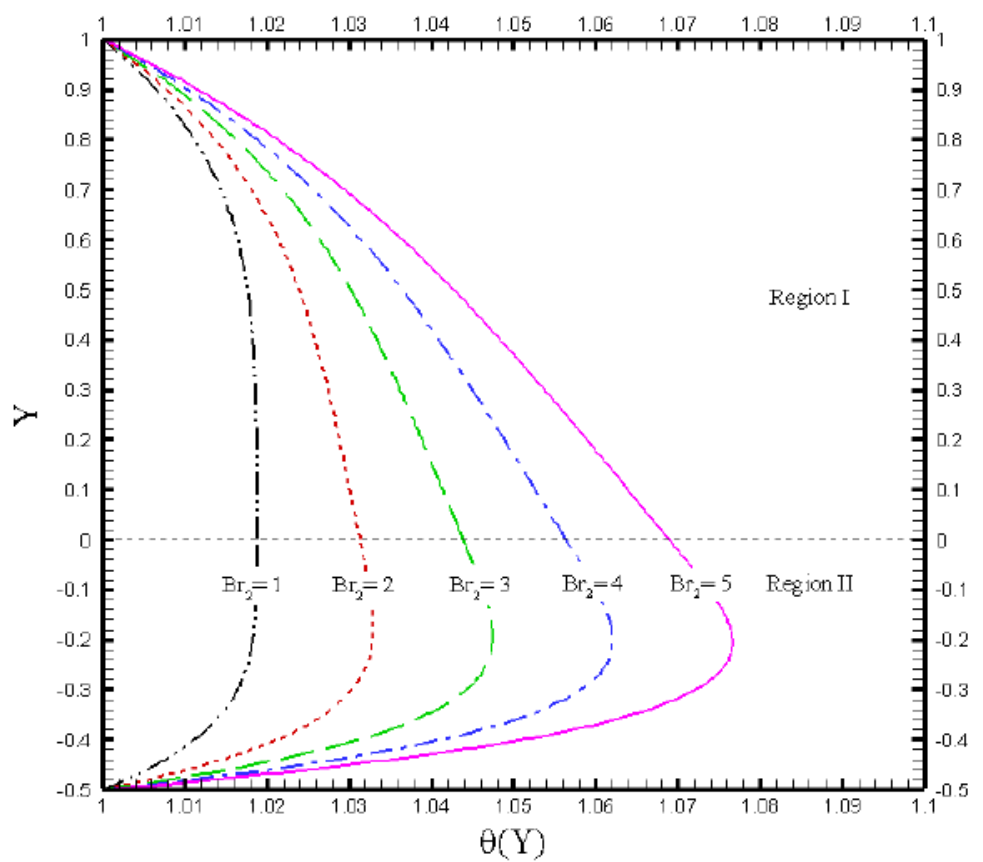

(b) For various values of $B r_{2}$ with $B r_{1}=1$

Fig.7 The dimensionless temperature profiles $\theta(Y)$ in the case of $\kappa_{1}=\kappa_{2}=1, H a_{1}=H a_{2}=1$, $N t=N b=0.1$ and $h=2$. 
Normally, the influence of viscous dissipation on the fluid flow is negligible in macro flow, therefore, viscous dissipation term often omitted under most circumstances. However, the effects of viscous dissipation in microscopic ones can be very strong, and the effect of viscous dissipation on temperature distribution can also be significant. Therefore, it can further lead to the flow being markedly changed in the microchannel. Fig.7(a) and Fig.7(b) exhibit the temperature distribution for different Brinkman numbers $\mathrm{Br}_{1}$ and $\mathrm{Br}_{2}$. Brinkman number is a dimensionless number representing the ratio of viscous heat generation to external heating. As seen in Fig.7(a), the dimensionless temperature increases with $B r_{1}$ increasing, especially in Region I of the microchannel. It is also shown in Fig.7(b) that $\mathrm{Br}_{2}$ has a significant effect on the dimensionless temperature in Region II of the microchannel. The temperature rise in the microchannel is mainly attributed to effect that the higher value of Brinkman number, the slower the conduction of heat generated by viscous dissipation and hence the greater the temperature rise.

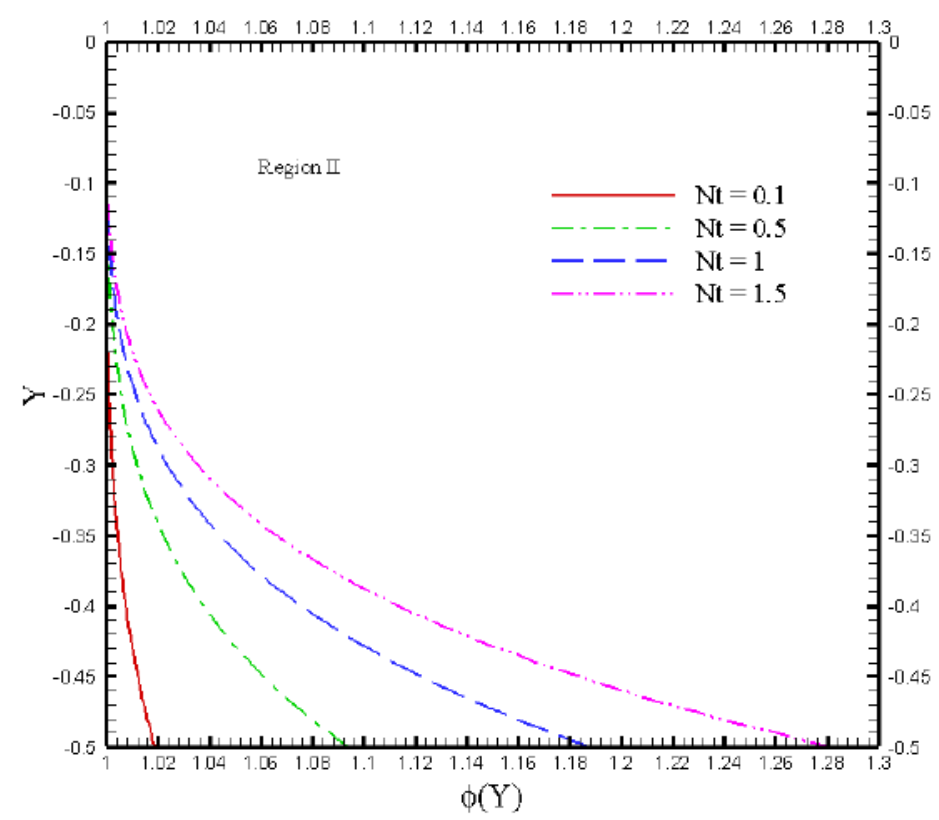

Fig.8 The nanoparticle volume fraction profiles $\phi(Y)$ for various values of $N t$ in the case of $\kappa_{1}=\kappa_{2}=1, H a_{1}=H a_{2}=1, N b=0.1, B r_{1}=B r_{2}=1$ and $h=2$.

The effects of thermophoresis parameter $N t$ on the nanoparticle volume fraction is depicted in Fig.8. It can be observed that increasing the value of the thermophoresis parameter, the nanoparticle volume fraction increases significantly in the vicinity of the 
lower wall. It is worth noting that the lower plate has the passive boundary condition due to the passively controlled nanofluid model in the present study. In other words, the concentration of nanoparticles on the wall is closely related to the temperature gradient near the wall. While the thermophoresis parameter is proportional to the temperature gradient. Therefore, the nanoparticle volume fraction on the lower wall varies with the change of $N t$.

The physical quantities such as the local skin friction, the local Nusselt number and the local Sherwood number are of importance in channel flow. The following section is mainly to examine the influence of physical parameters in region II on these physical quantities. The variation of the local Sherwood number with the thermophoresis parameter $N t$ for various values of the Brownian motion parameter $\mathrm{Nb}$ on the lower wall of the microchannel is shown in Fig.9. It is seen that the local Sherwood number increases monotonously as $N t$ increases. Also, it is obvious that the local Sherwood number decreases rapidly with $N b$ increasing. It is shows that $\mathrm{Nt}$ and $\mathrm{Nb}$ have a vital role in the mass transfer mechanism. That is to say, both the type and the size of the nanoparticles will affect the heat and mass transfer in the microchannel.

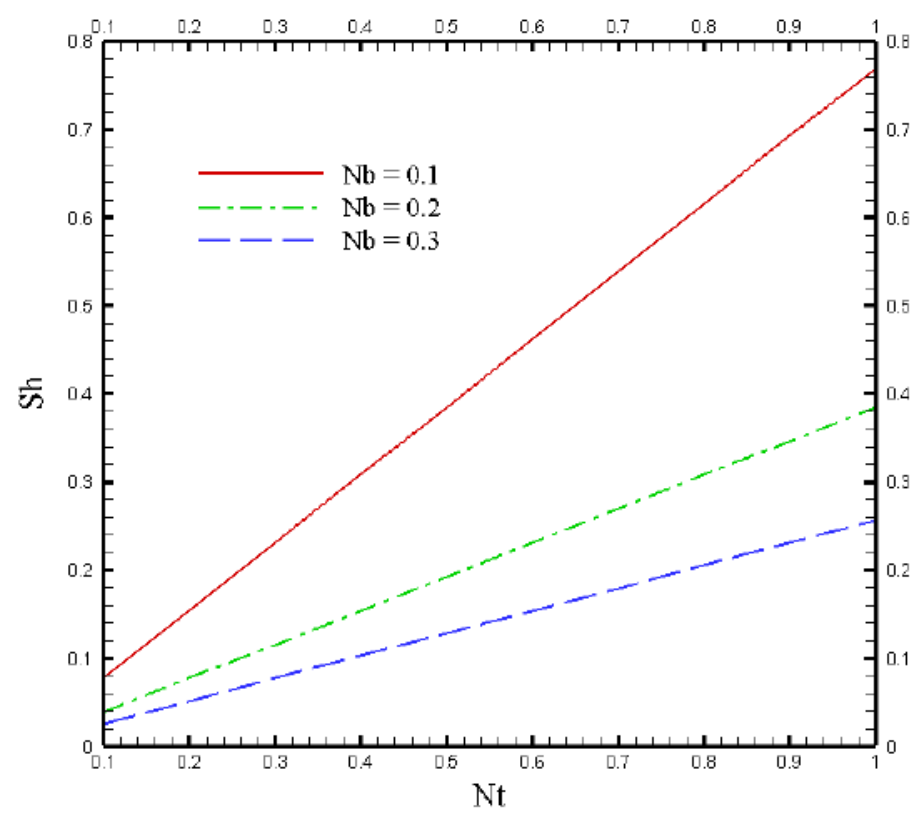

Fig.9 Variation of the local Sherwood number with the thermophoresis parameter $N t$ for various values of $N b$ in the case of $\kappa_{1}=\kappa_{2}=1, H a_{1}=H a_{2}=1, B r_{1}=B r_{2}=1$ and $h=2$. 
The influence of Hartmann number $\mathrm{Ha}_{2}$ on the local skin friction, the local Nusselt number and the local Sherwood number are examined in Fig.10 and Fig.11. It can be seen in Fig.10 and Fig.11 that the variation of $\mathrm{Ha}_{2}$ causes the different trends for these physical quantities. In Fig.10, the increase of $\mathrm{Ha}_{2}$ leads to a slight increase of the skin friction on the upper wall, but results in the decrease significantly of the skin friction on the lower wall. It is also observed that the effects of magnetic field parameter on Sherwood number is negligible. In Fig.11, the Nusselt number on the upper wall has a similar variation trend with the Sherwood number. The increase of $\mathrm{Ha}_{2}$ causes a slight increase of the Nusselt number on the lower wall. It is worth noting that, as $\mathrm{Ha}_{2}$ is considerably large, the values of the physical quantities close to certain constants, respectively. The influence of the parameter $\kappa_{2}$ on these physical quantities are also depicted in Fig.10 and Fig.11. It is easy to observe in Fig.10 that the increase of $\kappa_{2}$ leads to a slight increase of the skin friction and the Sherwood number on the lower wall, but causes a slight decrease of the skin friction on the upper wall. As seen in Fig.11, the increase of $\kappa_{2}$ makes the enhancement of the Nusselt number on the upper wall, while causes the reduction of the Nusselt number on the lower wall.

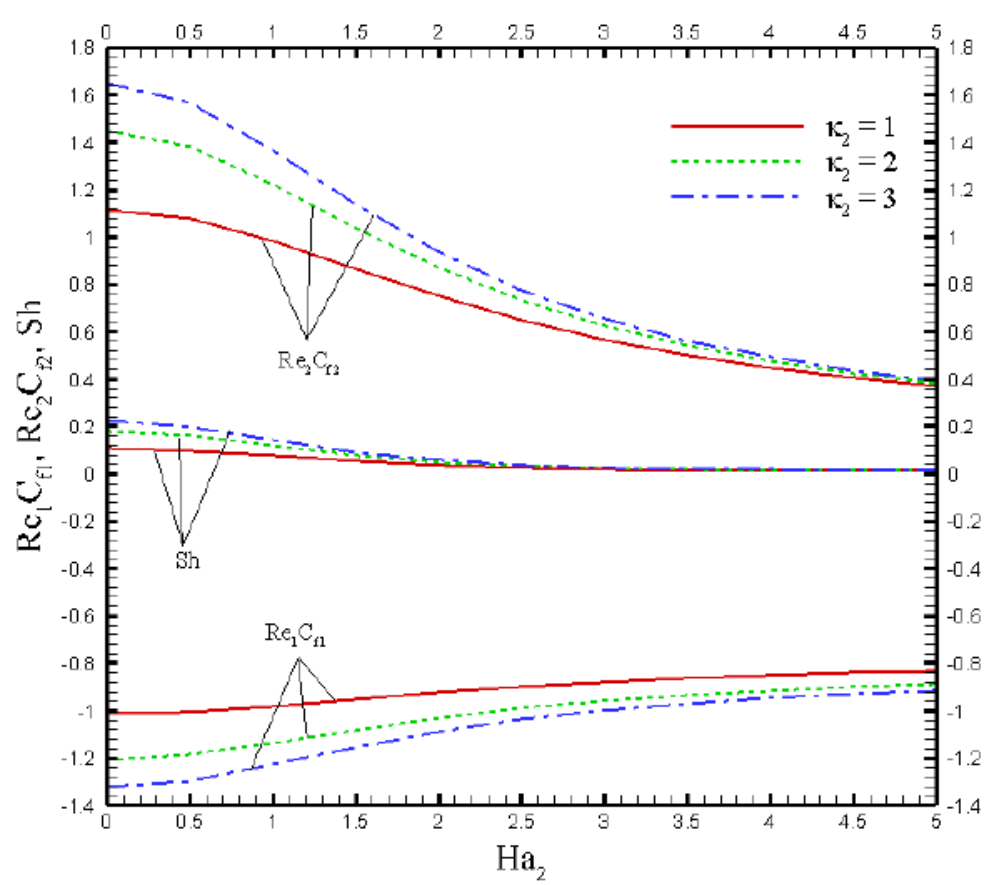

Fig.10 Variation of local skin friction and the local Sherwood number with $\mathrm{Ha}_{2}$ for various values of $\kappa_{2}$ in the case of $\kappa_{1}=1, H a_{1}=1, N t=N b=0.1, B r_{1}=B r_{2}=1$ and $h=2$. 


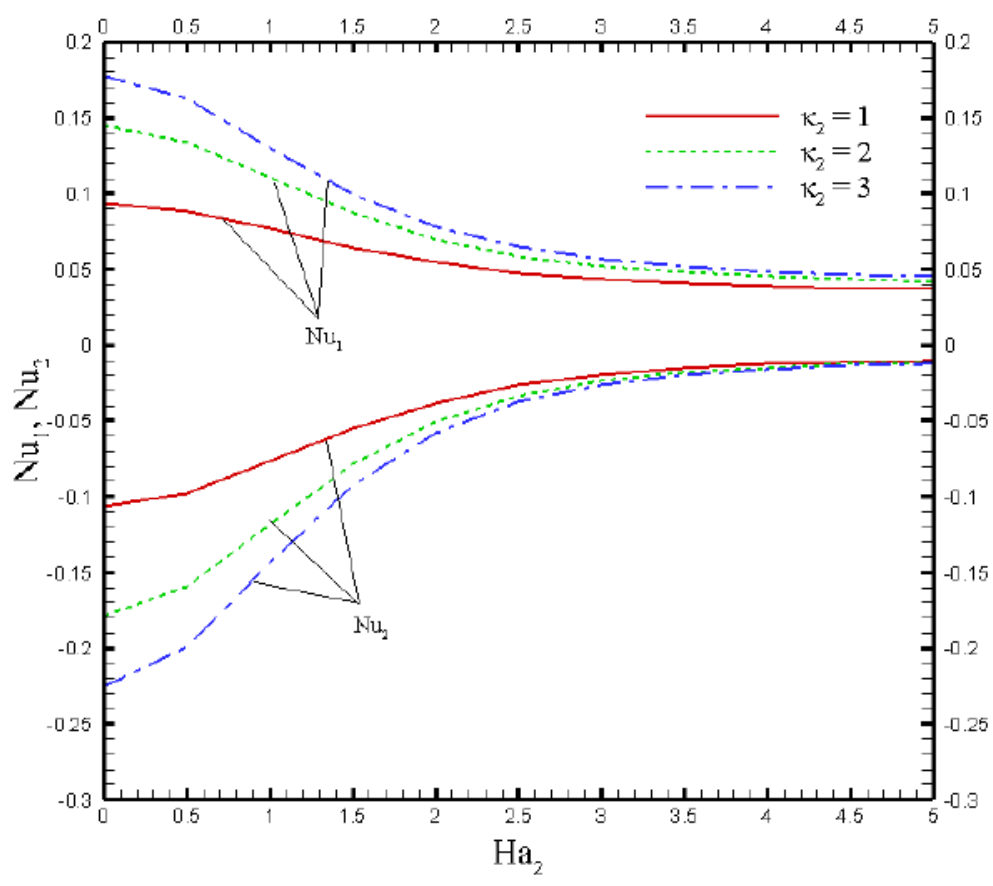

Fig.11 Variation of the local Nusselt number with $\mathrm{Ha}_{2}$ for various values of $\kappa_{2}$ in the case of $\kappa_{1}=1, H a_{1}=1, N t=N b=0.1, B r_{1}=B r_{2}=1$ and $h=2$.

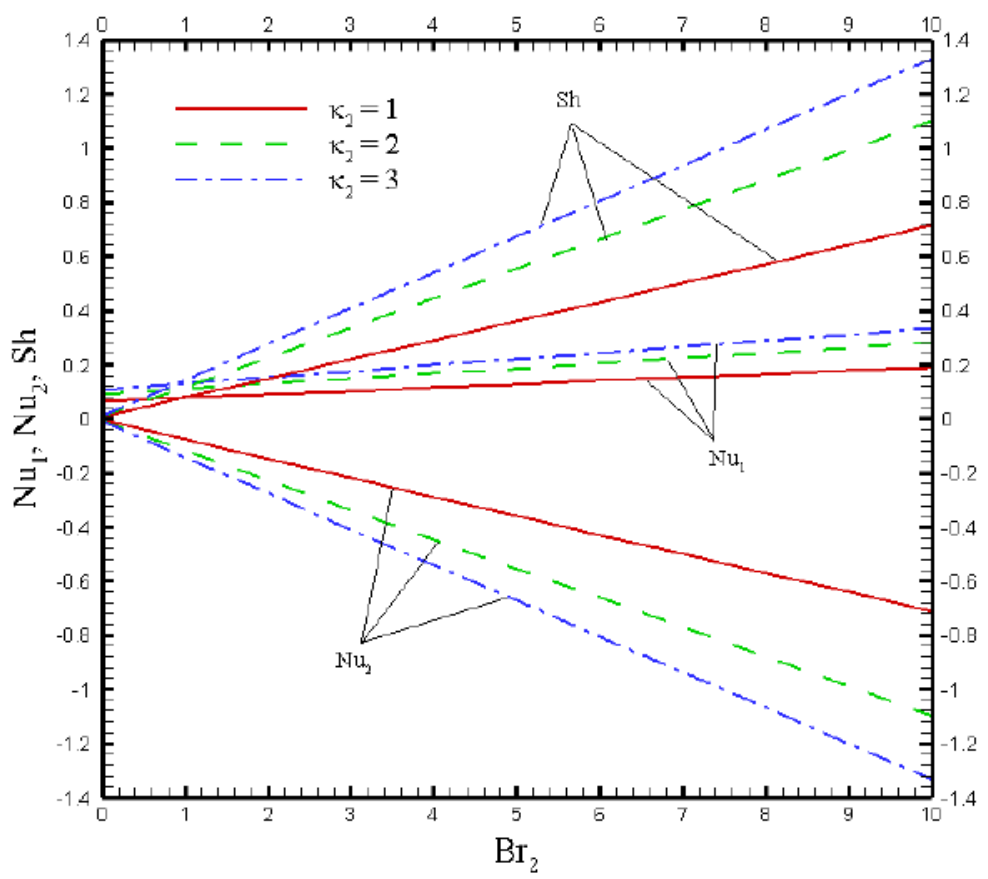

Fig.12 Variation of the local Nusselt number and the local Sherwood number with $\mathrm{Br}_{2}$ for various values of $\kappa_{2}$ in the case of $\kappa_{1}=1, B r_{1}=1, N t=N b=0.1, H a_{1}=H a_{2}=1$ and $h=2$. 
The influence of Brinkman number $\mathrm{Br}_{2}$ on the local Nusselt number and the local Sherwood number are presented in Fig.12. It is evident that the increase of $\mathrm{Br}_{2}$ leads to a significant increase of the local Sherwood number. The local Nusselt number on the upper wall increases slightly as $\mathrm{Br}_{2}$ increases, while the local Nusselt number on the lower wall decrease significantly as $\mathrm{Br}_{2}$ increases. It can also be observed that the local Nusselt number and the local Sherwood number have a linear relationship with Brinkman number $\mathrm{Br}_{2}$. Therefore, the Brinkman number can be used to predict the values of these physical quantities by using linear fitting to obtain their relationship. In Fig.12, the Nusselt number on the upper wall and the local Sherwood number increase with the enhancement of $\kappa_{2}$, while the Nusselt number on the lower wall reduces with the enhancement of $\kappa_{2}$, resemble trends observed in Fig.10 and Fig.11.

\section{Conclusions}

The heat and mass transfer of immiscible two-layer flows in the presence of the EDL and magnetic field have been investigated theoretically. Through nondimensionalization, the governing equations are transformed into nonlinear ordinary differential equations. The resulting coupled nonlinear equations are solved by homotopy analysis method. The results of electric potential, velocity, temperature and nanoparticle volume fraction are obtained and examined in detail. The main findings are as follows:

i). The explicit exact solutions of the electrostatic potential in both regions are obtained. Besides, the present results of electrostatic potential, velocity and temperature are consecutive and smooth in the whole region.

ii). The parameters $\kappa_{1}$ and $\kappa_{2}$ are the key factor to measure the EDL effects. The larger is the parameter $\kappa_{1}$ and $\kappa_{2}$, the thinner is the EDL on the upper wall and lower wall. For sufficiently large parameter $\kappa_{1}$ and $\kappa_{2}$, the EDL effects on the fluid motion and heat transfer can be ignored, thus the related quantities tend to be constants.

iii). Both the EDL effects and magnetic field can restrain the fluid flow in the microchannel. Therefore, the EDL effect and magnetic field can be applied for flow control in microchannels and microfluidic devices. 
In view of the HAM technique, we first define $\int_{0}^{1} U_{1} \Psi_{1} d Y, \int_{-1 / h}^{0} U_{2} \Psi_{2} d Y$ as below

$$
\int_{0}^{1} U_{1} \Psi_{1} d Y=m_{0}+\sum_{j=1}^{\infty} m_{j}, \int_{-1 / h}^{0} U_{2} \Psi_{2} d Y=n_{0}+\sum_{j=1}^{\infty} n_{j}
$$

then the expression for the functions $\Psi_{i}(Y), U_{i}(Y), \theta_{i}(Y)(i=1,2)$ and $\phi(Y)$ can be written as

$$
\Psi_{i}=\psi_{i, 0}+\sum_{j=1}^{\infty} \psi_{i, j}, U_{i}=u_{i, 0}+\sum_{j=1}^{\infty} u_{i, j}, \theta_{i}=\theta_{i, 0}+\sum_{j=1}^{\infty} \theta_{i, j}, \phi=\phi_{0}+\sum_{j=1}^{\infty} \phi_{j} .
$$

In the solution process of the HAM, the $m$ th order HAM deformation equations can be written as

$$
\begin{gathered}
\psi_{1, m}^{\prime \prime}-\chi_{m} \psi_{1, m-1}^{\prime \prime}=\hbar_{\psi_{1}} R_{\psi_{1}, m}, \quad \psi_{2, m}^{\prime \prime}-\chi_{m} \psi_{2, m-1}^{\prime \prime}=\hbar_{\psi_{2}} R_{\psi_{2}, m}, \\
u_{1, m}^{\prime \prime}-\chi_{m} u_{1, m-1}^{\prime \prime}=\hbar_{u_{1}} R_{u_{1}, m}, \quad u_{2, m}^{\prime \prime}-\chi_{m} u_{2, m-1}^{\prime \prime}=\hbar_{u_{2}} R_{u_{2}, m}, \\
\theta_{1, m}^{\prime \prime}-\chi_{m} \theta_{1, m-1}^{\prime \prime}=\hbar_{\theta_{1}} R_{\theta_{1}, m}, \quad \theta_{2, m}^{\prime \prime}-\chi_{m} \theta_{2, m-1}^{\prime \prime}=\hbar_{\theta_{2}} R_{\theta_{2}, m}, \\
\phi_{m}^{\prime \prime}-\chi_{m} \phi_{m-1}^{\prime \prime}=\hbar_{\phi} R_{\phi, m},
\end{gathered}
$$

The corresponding boundary conditions are 


$$
\begin{aligned}
& \psi_{1, m}(1)=0, \psi_{2, m}\left(-\frac{1}{h}\right)=0, \psi_{1, m}(0)=\hat{z} \psi_{2, m}(0), \psi_{1, m}^{\prime}(0)=\frac{\hat{z}}{\varepsilon} \psi_{2, m}^{\prime}(0), \\
& u_{1, m}(1)=0, u_{2, m}\left(-\frac{1}{h}\right)=0, u_{1, m}(0)=u_{2, m}(0), u_{1, m}^{\prime}(0)=\frac{1}{\mu} u_{2, m}^{\prime}(0), \\
& \theta_{1, m}(1)=0, \theta_{2, m}\left(-\frac{1}{h}\right)=0, \theta_{1, m}(0)=\delta \theta_{2, m}(0), \theta_{1, m}^{\prime}(0)=\frac{\delta}{k_{f}} \theta_{2, m}^{\prime}(0), \\
& \phi_{m}(0)=0, N b \phi_{m}^{\prime}\left(-\frac{1}{h}\right)+N t \theta_{2, m}^{\prime}\left(-\frac{1}{h}\right)=0,
\end{aligned}
$$

where $\hbar_{\psi_{i}}, \hbar_{u_{i}}, \hbar_{\theta_{i}}(i=1,2)$ and $\hbar_{\phi}$ are the HAM auxiliary parameters, which provide us a simple way to adjust and control the convergence of the HAM analytical approximations. Also, $R_{\psi_{i}, m}, R_{u_{i}, m}, R_{\theta_{i}, m}(i=1,2), R_{\phi, m}$, and $\chi_{m}$, are defined, respectively, as

$$
\begin{aligned}
& R_{\psi_{1}, m}=\psi_{1, m-1}^{\prime \prime}-\kappa_{1}^{2} \psi_{1, m-1}, \\
& R_{\psi_{2}, m}=\psi_{2, m-1}^{\prime \prime}-h^{2} \kappa_{2}^{2} \psi_{2, m-1}, \\
& R_{u_{1}, m}=u_{1, m-1}^{\prime \prime}+P_{1}\left(1-\chi_{m}\right)-4 G_{1} G_{3} \sum_{j=0}^{m-1} m_{j} \psi_{1, m-1-j}-H a_{1}^{2} u_{1, m-1}, \\
& R_{u_{2}, m}=u_{2, m-1}^{\prime \prime}+h^{2} P_{2}\left(1-\chi_{m}\right)-4 h^{3} G_{2} G_{4} \sum_{j=0}^{m-1} n_{j} \psi_{2, m-1-j}-h^{2} H a_{2}^{2} u_{2, m-1}, \\
& R_{\theta_{1}, m}=\theta_{1, m-1}^{\prime \prime}+B r_{1} \sum_{j=0}^{m-1} u_{1, j}^{\prime} u_{1, m-1-j}^{\prime}, \\
& R_{\theta_{2}, m}=\theta_{2, m-1}^{\prime \prime}+N b \sum_{j=0}^{m-1} \phi_{j}^{\prime} \theta_{2, m-1-j}^{\prime}+N t \sum_{j=0}^{m-1} \theta_{2, j}^{\prime} \theta_{2, m-1-j}^{\prime}+B r_{2} \sum_{j=0}^{m-1} u_{2, j}^{\prime} u_{2, m-1-j}^{\prime}, \\
& R_{\phi, m}=\phi_{m-1}^{\prime \prime}+\frac{N t}{N b} \theta_{2, m-1}^{\prime \prime},
\end{aligned}
$$

where

$$
\chi_{m}= \begin{cases}0, & m \leq 1 \\ 1, & m>1\end{cases}
$$

The solutions of Eqs.(61)-(64) are expressed as

$$
\begin{aligned}
& \psi_{1, m}=\psi_{1}^{*}+\chi_{m} \psi_{1, m-1}+C_{1, m}+C_{2, m} Y, \\
& \psi_{2, m}=\psi_{2}^{*}+\chi_{m} \psi_{2, m-1}+C_{3, m}+C_{4, m} Y,
\end{aligned}
$$




$$
\begin{aligned}
& u_{1, m}=u_{1}^{*}+\chi_{m} u_{1, m-1}+C_{5, m}+C_{6, m} Y, \\
& u_{2, m}=u_{2}^{*}+\chi_{m} u_{2, m-1}+C_{7, m}+C_{8, m} Y, \\
& \theta_{1, m}=\theta_{1}^{*}+\chi_{m} \theta_{1, m-1}+C_{9, m}+C_{10, m} Y, \\
& \theta_{2, m}=\theta_{2}^{*}+\chi_{m} \theta_{2, m-1}+C_{11, m}+C_{12, m} Y, \\
& \phi_{m}=\phi^{*}+\chi_{m} \phi_{m-1}+C_{13, m}+C_{14, m} Y,
\end{aligned}
$$

where

$$
\begin{aligned}
\psi_{1}^{*} & =\iint \hbar_{\psi_{1}} R_{\psi_{1}, m} d Y d Y, \psi_{2}^{*}=\iint \hbar_{\psi_{2}} R_{\psi_{2}, m} d Y d Y, \\
u_{1}^{*} & =\iint \hbar_{u_{1}} R_{u_{1}, m} d Y d Y, u_{2}^{*}=\iint \hbar_{u_{2}} R_{u_{2}, m} d Y d Y, \\
\theta_{1}^{*} & =\iint \hbar_{\theta_{1}} R_{\theta_{1}, m} d Y d Y, \theta_{2}^{*}=\iint \hbar_{\theta_{2}} R_{\theta_{2}, m} d Y d Y, \\
\phi^{*} & =\iint \hbar_{\phi} R_{\phi, m} d Y d Y .
\end{aligned}
$$

The integral constants $C_{i, m}(i=1,2, \cdots, 14)$ in Eqs.(67)-(73) are determined by the boundary conditions (65). Therefore, all solutions of the problem are obtained.

The initial guess $\psi_{0}(Y), u_{0}(Y), \theta_{0}(Y)$ and $\phi_{0}(Y)$ should be chosen properly. Based on the boundary conditions (27), (28), (34) and (52), the initial guess values in the present study are chosen as follows

$$
\begin{aligned}
& \psi_{1,0}(Y)=\bar{\zeta}_{1} Y^{2}, \psi_{2,0}(Y)=\bar{\zeta}_{2} h^{2} Y^{2} \\
& u_{1,0}(Y)=Y^{2}+\frac{1-h^{2}}{h(h+\mu)} Y-\frac{1+h \mu}{h(h+\mu)}, u_{2,0}(Y)=Y^{2}+\frac{\left(1-h^{2}\right) \mu}{h(h+\mu)} Y-\frac{1+h \mu}{h(h+\mu)}, \\
& \theta_{1,0}(Y)=\frac{h(1-\delta)}{h+k_{f}} Y+\frac{\delta h+k_{f}}{h+k_{f}}, \theta_{2,0}(Y)=\frac{h k_{f}(1-\delta)}{\delta\left(h+k_{f}\right)} Y+\frac{\delta h+k_{f}}{\delta\left(h+k_{f}\right)}, \\
& \phi_{0}(Y)=\frac{N t}{N b} \frac{h k_{f}(\delta-1)}{\delta\left(h+k_{f}\right)} Y+1 .
\end{aligned}
$$

In the HAM solution procedure, the integral terms $\int_{0}^{1} u_{1,0} \psi_{1,0} d Y$ and $\int_{-1 / h}^{0} u_{2,0} \psi_{2,0} d Y$ can be obtained using the initial guess values. Therefore, all the solution series for the integral terms can be obtained from $m=1,2,3, \cdots$. 


\section{Reference}

Abd Elmaboud, Y., Abdelsalam, S.I., Mekheimer, K.S. and Vafai, K. (2019), "Electromagnetic flow for two-layer immiscible fluids", Engineering Science and Technology, an International Journal, Vol. 22 No.1, pp.237-248.

Buongiorno, J. (2006), "Convective transport in nanofluids", Journal of heat transfer, Vol.128 No. 3, pp.240-250.

Choi, S. U. and Eastman, J. A. (1995), Enhancing thermal conductivity of fluids with nanoparticles. No. ANL/MSD/CP-84938; CONF-951135-29. Argonne National Lab., IL (United States).

Darabi, J. and Ekula, K. (2003), "Development of a chip-integrated micro cooling device”, Microelectronics Journal, Vol. 34 No. 11, pp.1067-1074.

Del Arco, E.C., Extremet, G.P. and Sani, R.L. (1991), Thermocapillary convection in a two-layer fluid system with flat interface", Advances in Space Research, Vol. 11 No.7, pp.129-132.

Donaldson, L. (2009), "Small and powerful nuclear battery developed", materials today, Vol. 12 No. 11, p.10.

Guo, Z. Y. and Li, Z. X. (2003), "Size effect on single-phase channel flow and heat transfer at microscale", International Journal of Heat and Fluid Flow, Vol. 24 No. 3, pp.284-298.

Heris, S. Z., Etemad, S. G. and Esfahany, M. N. (2006), "Experimental investigation of oxide nanofluids laminar flow convective heat transfer", International communications in heat and mass transfer, Vol. 33 No. 4, pp.529-535.

Hunter, R. J. (1981), Zeta potential in colloid science: principles and applications. Academic press, New York.

Jing, D., Pan, Y. and Wang, X. (2017), "Joule heating, viscous dissipation and convective heat transfer of pressure-driven flow in a microchannel with surface chargedependent slip", International Journal of Heat and Mass Transfer, Vol. 108, pp.13051313.

Karimipour, A., Nezhad, A. H., D'orazio, A., Esfe, M. H., Safaei, M. R. and Shirani, E. (2015), "Simulation of copper-water nanofluid in a microchannel in slip flow regime using the lattice Boltzmann method", European Journal of MechanicsB/Fluids, Vol.49, pp.89-99.

Kulasegaram, S., Bonet, J., Lewis, R. W. and Profit, M. (2004), “A variational formulation based contact algorithm for rigid boundaries in two-dimensional SPH applications", Computational Mechanics, Vol. 33 No.4, pp.316-325.

Kuznetsov, A. V. and Nield, D. A. (2010), "Natural convective boundary-layer flow of a nanofluid past a vertical plate", International Journal of Thermal Sciences, Vol.49 No.2, pp.243-247.

Kuznetsov, A. V. and Nield, D. A. (2013), "The Cheng-Minkowycz problem for natural convective boundary layer flow in a porous medium saturated by a nanofluid: a revised model", International Journal of Heat and Mass Transfer, Vol.65, pp.682-685.

Lewis, R. W., Morgan, K., Thomas, H. R. and Seetharamu, K. N. (1996), The finite 
element method in heat transfer analysis. John Wiley and Sons.

Lewis, R. W. and Ghafouri, H. R. (1997), "A novel finite element double porosity model for multiphase flow through deformable fractured porous media", International journal for numerical and analytical methods in geomechanics, Vol21 No.11, pp.789-816.

Lewis, R. W., Nithiarasu, P. and Seetharamu, K. N. (2004). Fundamentals of the finite element method for heat and fluid flow. John Wiley and Sons.

Li, H., Wong, T. N. and Nguyen, N. T. (2010), "Time-dependent model of mixed electroosmotic/pressure-driven three immiscible fluids in a rectangular microchannel", International journal of heat and mass transfer, Vol.53 No.4, pp.772-785.

Liao, S. (2012), Homotopy analysis method in nonlinear differential equations. Higher education press, Beijing.

Mala, G. M., Li D. and Dale, J. D. (1997), "Heat transfer and fluid flow in microchannels", International journal of heat and mass transfer, Vol. 40 No. 13, pp.3079-3088.

Mala, G. M. and Li, D. (1999), "Flow characteristics of water in microtubes", International journal of heat and fluid flow, Vol. 20 No. 2, pp.142-148.

Manikandan, S. and Rajan, K. S. (2015), "MgO-Therminol 55 nanofluids for efficient energy management: analysis of transient heat transfer performance", Energy, Vol.88, pp.408-416.

Moh'd A, A.N. and Al-Dafaie, A.M.A. (2014), "Using nanofluids in enhancing the performance of a novel two-layer solar pond", Energy, Vol. 68 No.4, pp.318-326.

Nithiarasu, P., Lewis, R. W. and Seetharamu, K. N. (2016). Fundamentals of the finite element method for heat and mass transfer. John Wiley and Sons.

Qi, C. and Ng, C. O. (2018), "Electroosmotic flow of a two-layer fluid in a slit channel with gradually varying wall shape and zeta potential", International Journal of Heat and Mass Transfer, Vol.119, pp.52-64.

Rahman, M. M., Rosca, A. V. and Pop, I. (2015), "Boundary layer flow of a nanofluid past a permeable exponentially shrinking surface with convective boundary condition using Buongiorno's model", International Journal of Numerical Methods for Heat and Fluid Flow. Vol. 25 No.2.

Redapangu, P. R., Chandra Sahu, K. and Vanka, S. P. (2012), “A study of pressuredriven displacement flow of two immiscible liquids using a multiphase lattice Boltzmann approach", Physics offluids, Vol. 24 No.10, p.102110.

Ren, L., Qu, W. and Li, D. (2001), "Interfacial electrokinetic effects on liquid flow in microchannels", International journal of heat and mass transfer, Vol. 44 No.16, pp.3125-3134.

Ren, C. L. and Li, D. (2005), "Improved understanding of the effect of electrical double layer on pressure-driven flow in microchannels", Analytica Chimica Acta, Vol. 531 No. 1, pp.15-23.

Rohni, A. M., Ahmad, S., Ismail, A. I. M. and Pop, I. (2013), "Flow and heat transfer over an unsteady shrinking sheet with suction in a nanofluid using Buongiorno's model", International Communications in Heat and Mass Transfer, Vol.43, pp.75-80. 
Sheremet, M. A. and Pop, I. (2014), "Natural convection in a square porous cavity with sinusoidal temperature distributions on both side walls filled with a nanofluid: Buongiorno's mathematical model", Transport in Porous Media, Vol.105 No. 2, pp.411-429.

Shit, G. C., Mondal, A., Sinha, A. and Kundu, P.K. (2016), "Two-layer electroosmotic flow and heat transfer in a hydrophobic micro-channel with fluid-solid interfacial slip and zeta potential difference." Colloids and Surfaces A: Physicochemical and Engineering Aspects, Vol. 506, pp.535-549.

Srinivas, B. (2016), "Electroosmotic flow of a power law fluid in an elliptic microchannel", Colloids and surfaces A: Physicochemical and Engineering Aspects, Vol. 492, pp.144-151.

Wang, B. X. and Peng, X. F. (1994), "Experimental investigation on liquid forcedconvection heat transfer through microchannels", International Journal of Heat and Mass Transfer, Vol. 37, pp.73-82.

Wang, X. J., Li, X. F., Xu, Y. H. and Zhu, D. S. (2014), “Thermal energy storage characteristics of $\mathrm{Cu}-\mathrm{H}_{2} \mathrm{O}$ nanofluids", Energy, Vol.78, pp.212-217

Williams, W., Buongiorno, J. and HU, L. W. (2008), "Experimental investigation of turbulent convective heat transfer and pressure loss of alumina/water and zirconia/water nanoparticle colloids (nanofluids) in horizontal tubes", Journal of heat transfer, Vol.130 No.4, p.042412.

Xie, Z. Y. and Jian, Y. J. (2017), "Entropy generation of two-layer magnetohydrodynamic electroosmotic flow through microparallel channels", Energy, Vol.139, pp.1080-1093.

Xuan, Y. and Li, Q. (2003), "Investigation on convective heat transfer and flow features of nanofluids", Journal of heat transfer, Vol.125 No. 1, pp.151-155.

You, X. Y. and Guo, L. X. (2010), "Analysis of EDL effects on the flow and flow stability in microchannels", Journal of Hydrodynamics, Vol. 22 No.5, pp.725-731.

$\mathrm{Yu}, \mathrm{Q} ., \mathrm{Xu}, \mathrm{H}$. and Liao, S. (2018), "Analysis of mixed convection flow in an inclined lid-driven enclosure with Buongiorno's nanofluid model", International Journal of Heat and Mass Transfer, Vol. 126, pp.221-236.

Zhao, Q., Xu, H. and Tao, L. (2018), "Nanofluid flow and heat transfer in a microchannel with interfacial electrokinetic effects", International Journal of Heat and Mass Transfer, Vol.124, pp.158-167.

Zheng, J. and Jian, Y. (2018), "Rotating electroosmotic flow of two-layer fluids through a microparallel channel", International Journal of Mechanical Sciences, Vol.136, pp.293-302. 


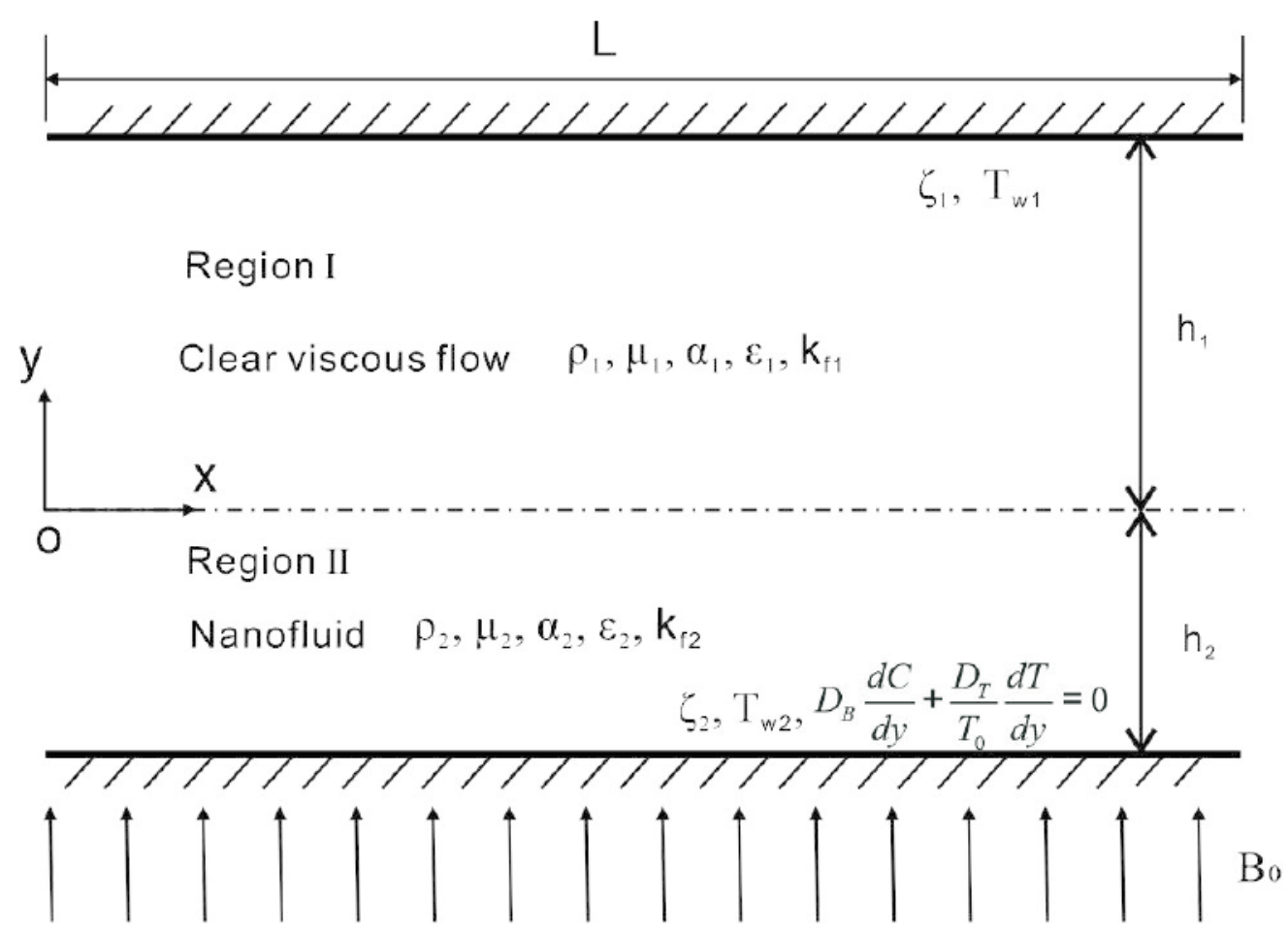

Fig1. Schematic of the physical model

$82 \times 58 \mathrm{~mm}(300 \times 300$ DPI $)$ 


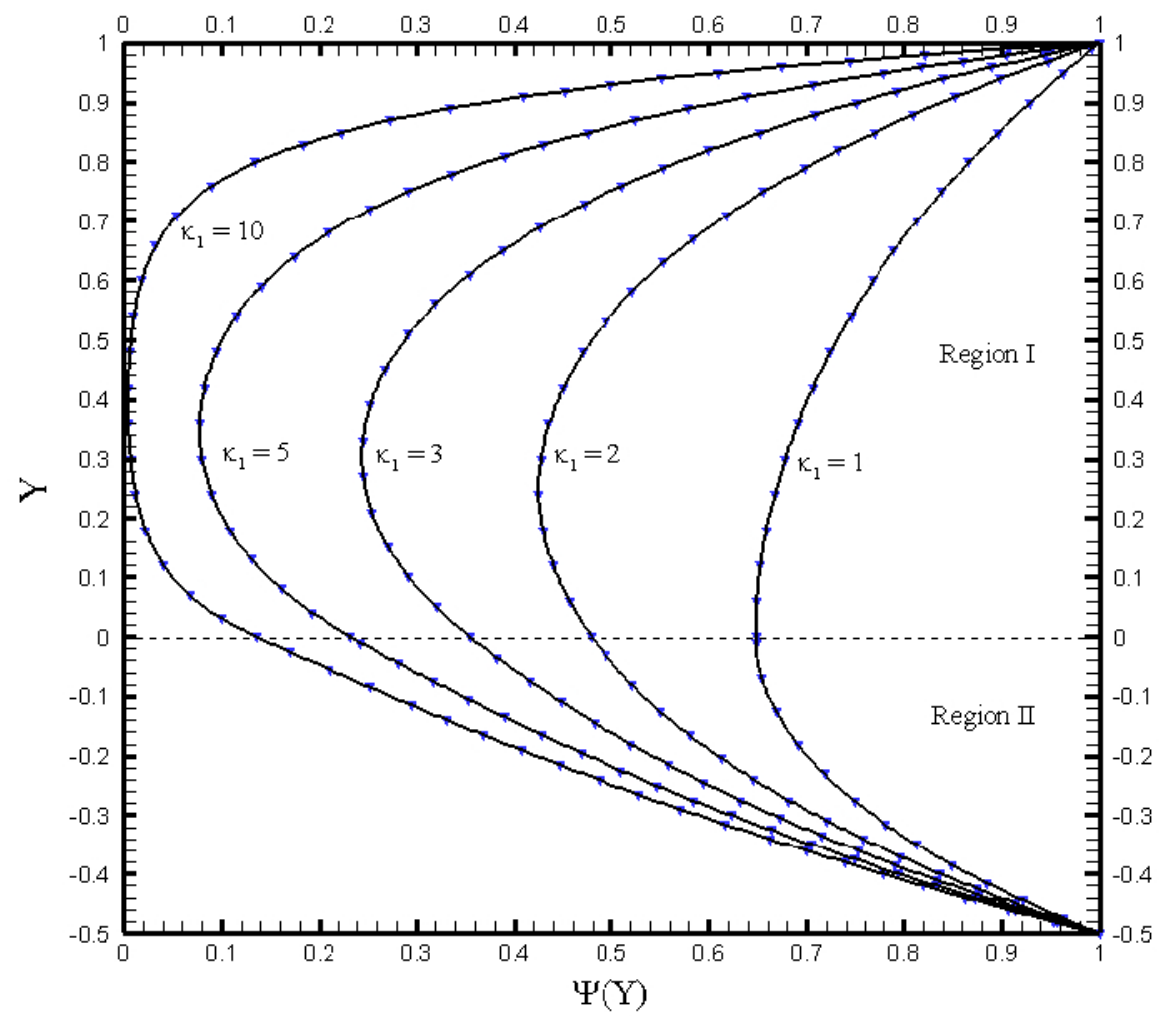

(a) For various values of $k 1$ with $k 2=1$ 


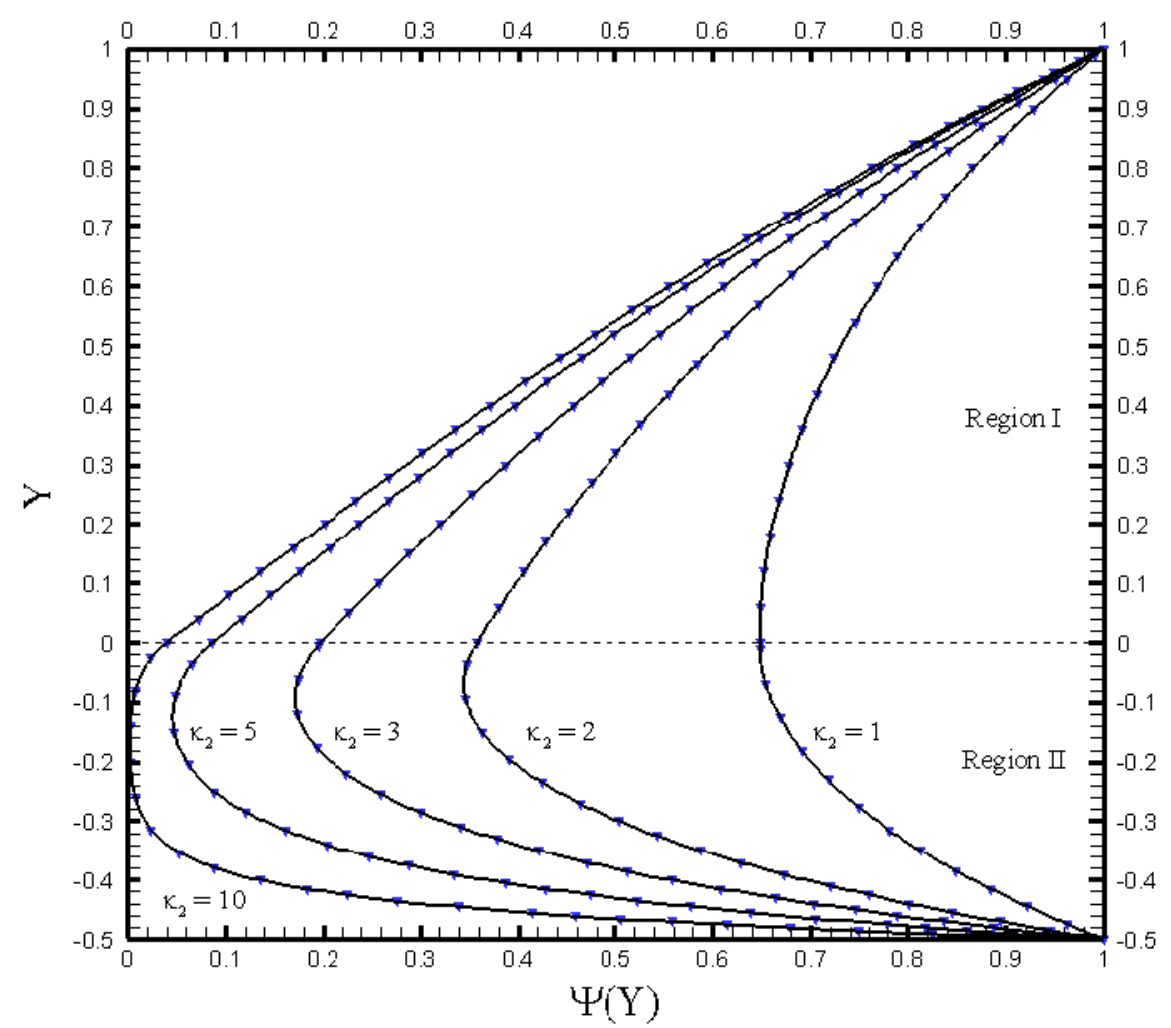

(b) For various values of $k 2$ with $k 1=1$

Fig.2 The electrical potential $\Psi(Y)$ with $\varepsilon=z=1, \varsigma 1=\varsigma 2=1, h=2$. Line: analytical solutions given by Eqs.(29) and (30); Triangle symbols: HAM solutions. 


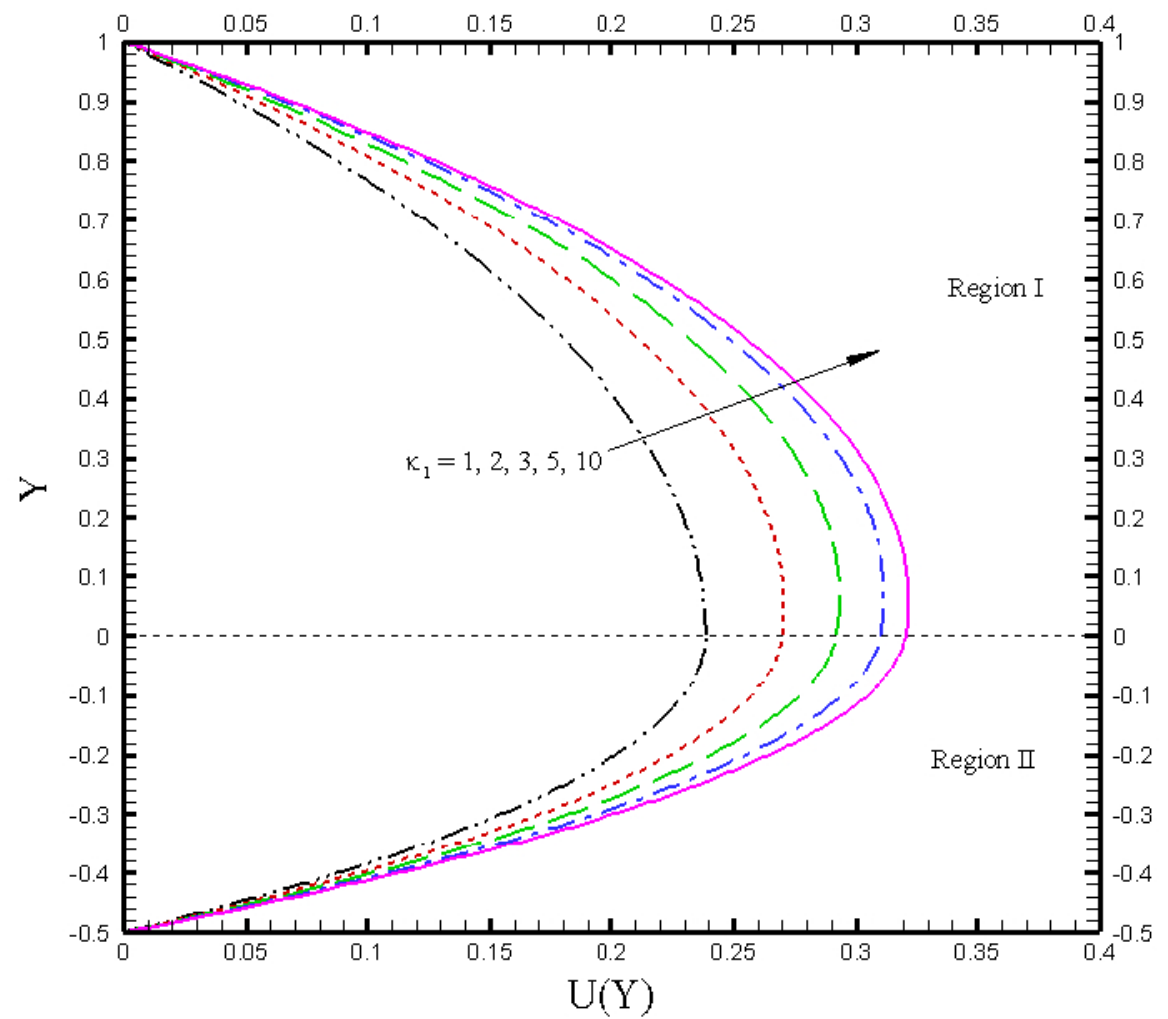

(a) For various values of $k 1$ with $k 2=1$ 


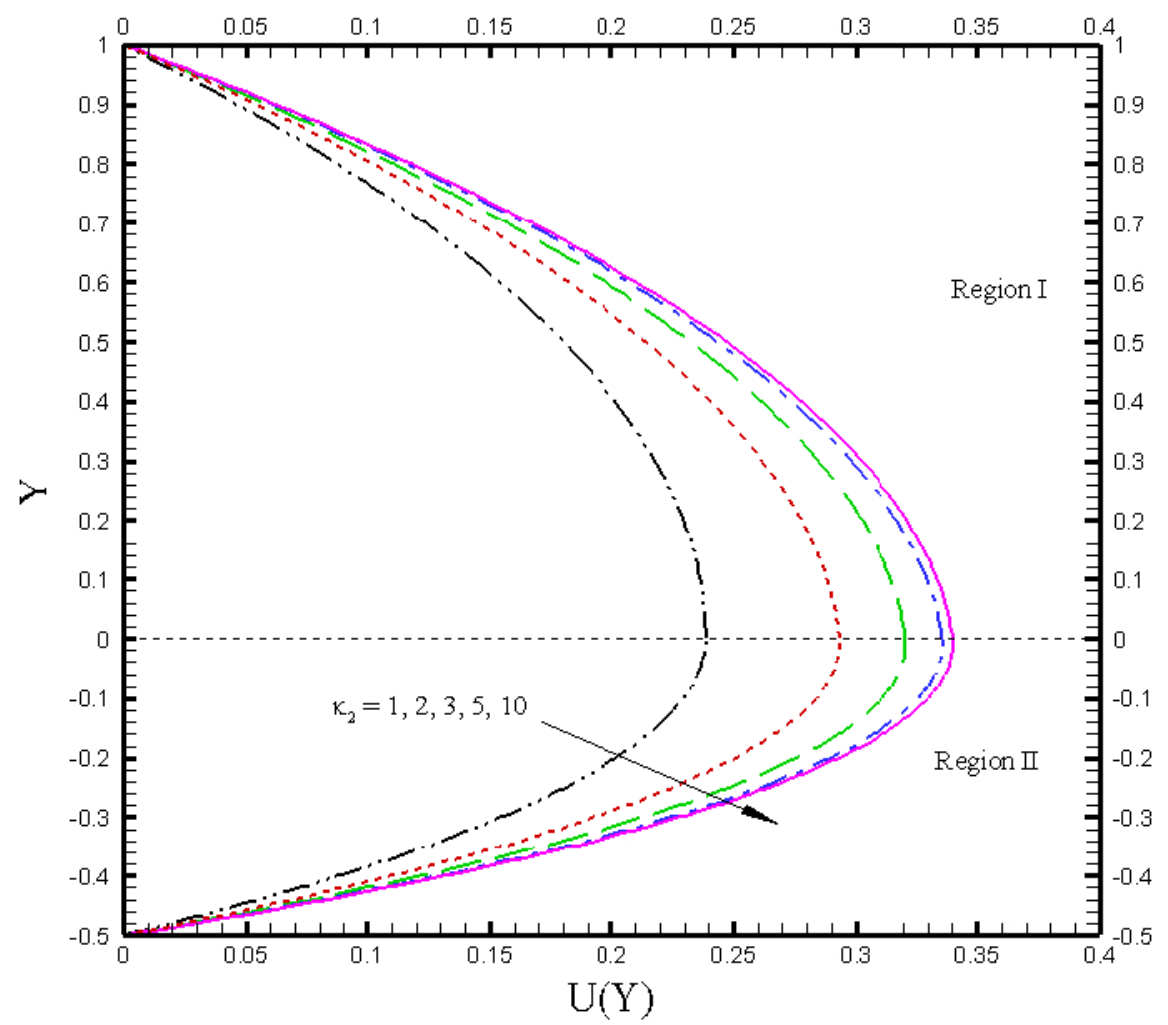

(b) For various values of $k 2$ with $k 1=1$

Fig. 3 The dimensionless velocity profiles $\mathrm{U}(\mathrm{Y})$ in the case of $\mathrm{G} 1=\mathrm{G} 2=\mathrm{G} 3=\mathrm{G} 4=1, \mathrm{P} 1=\mathrm{P} 2=1, \mathrm{Ha} 1=\mathrm{Ha} 2=1$ and $\mathrm{h}=2$. 


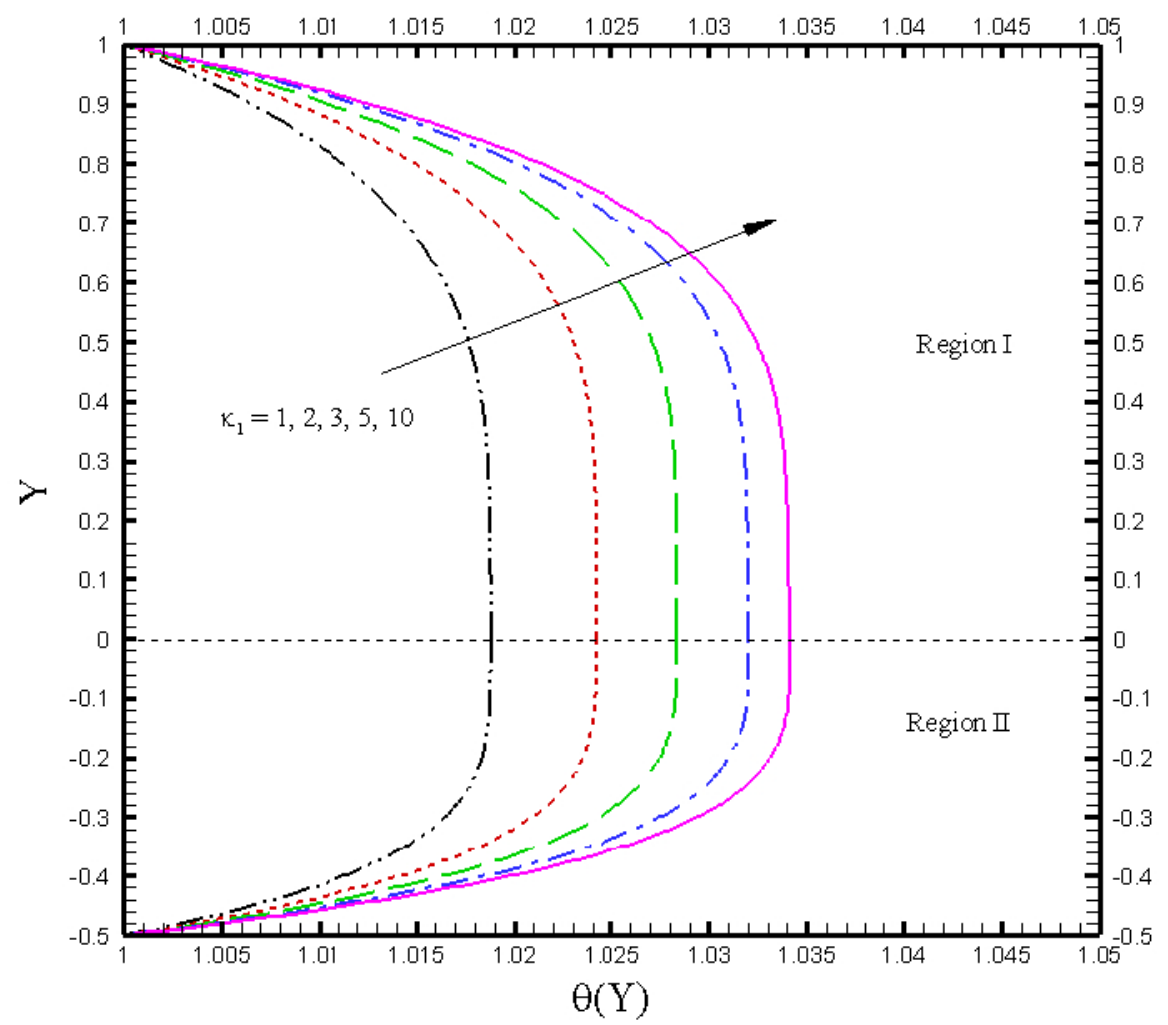

(a) For various values of $\mathrm{k} 1$ with $\mathrm{k} 2=1$ 


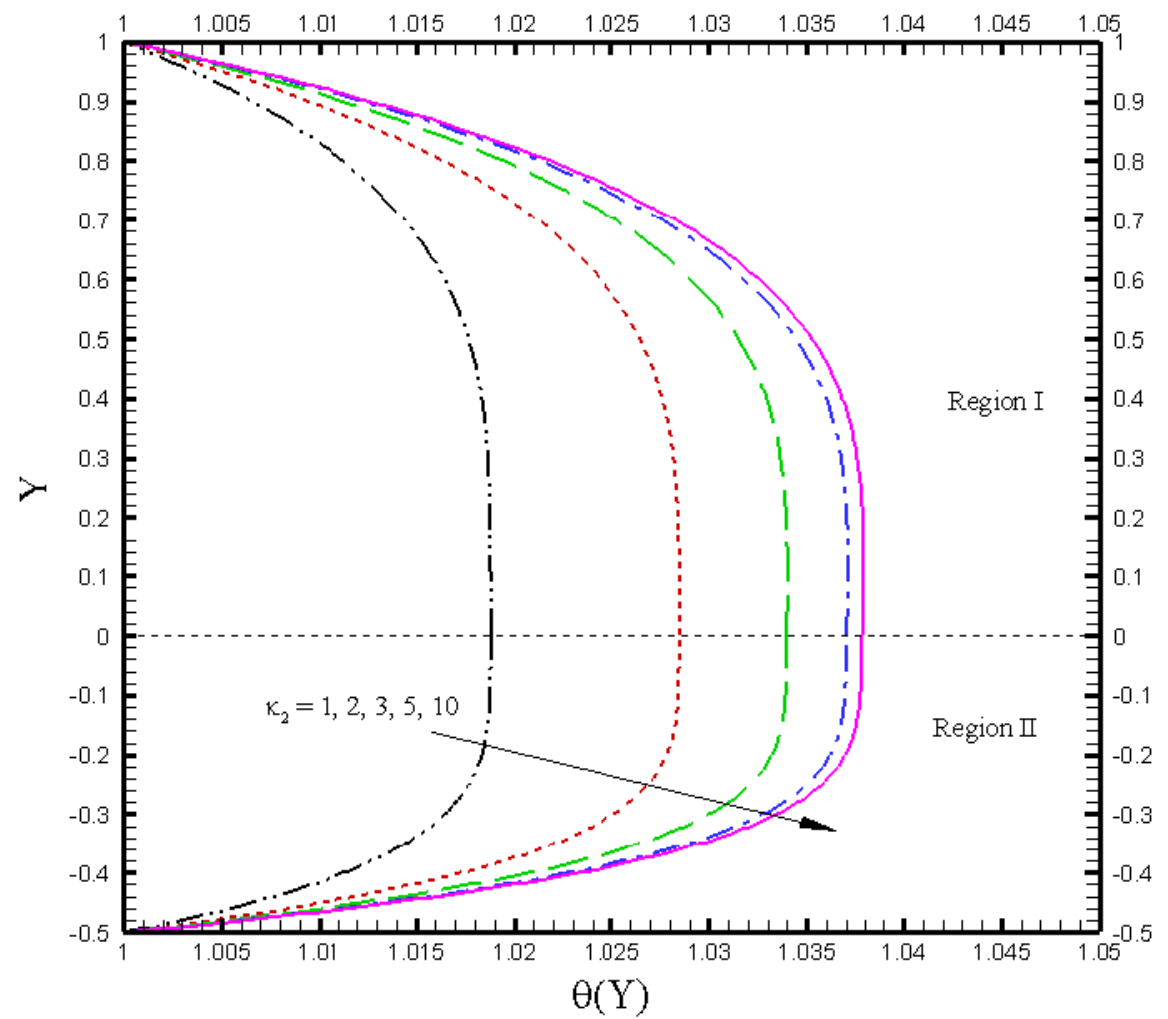

(b) For various values of $k 2$ with $k 1=1$

Fig.4 The dimensionless temperature profiles $\theta(\mathrm{Y})$ in the case of $\mathrm{Ha} 1=\mathrm{Ha} 2=1, \mathrm{Nt}=\mathrm{Nb}=0.1, \mathrm{Br} 1=\mathrm{Br} 2=1$ and $h=2$. 


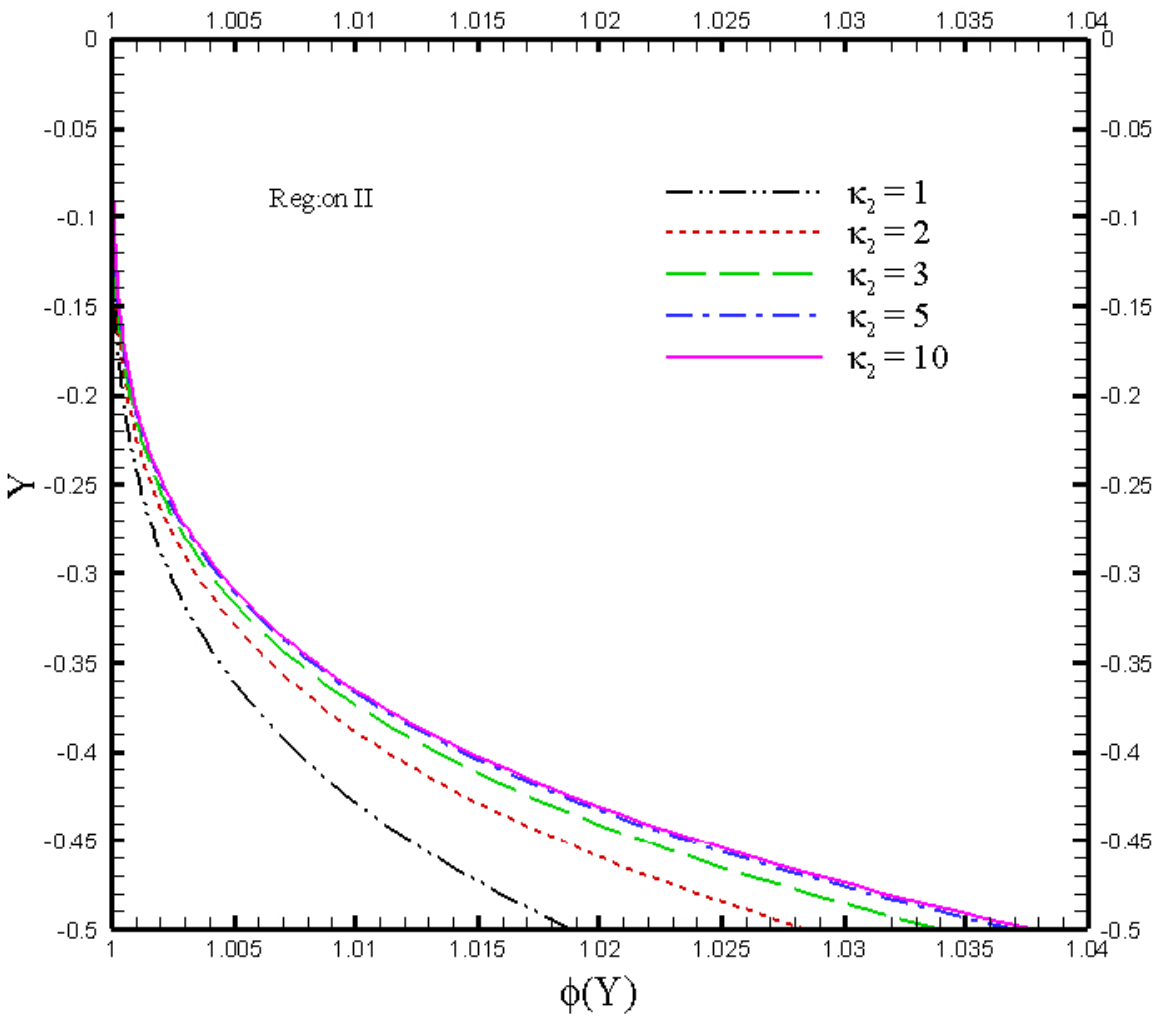

Fig. 5 The nanoparticle volume fraction profiles $\Phi(Y)$ for various values of $k 2$ in the case of $k 1=1$, $\mathrm{Ha} 1=\mathrm{Ha} 2=1, \mathrm{Nt}=\mathrm{Nb}=0.1, \mathrm{Br} 1=\mathrm{Br} 2=1$ and $\mathrm{h}=2$. 


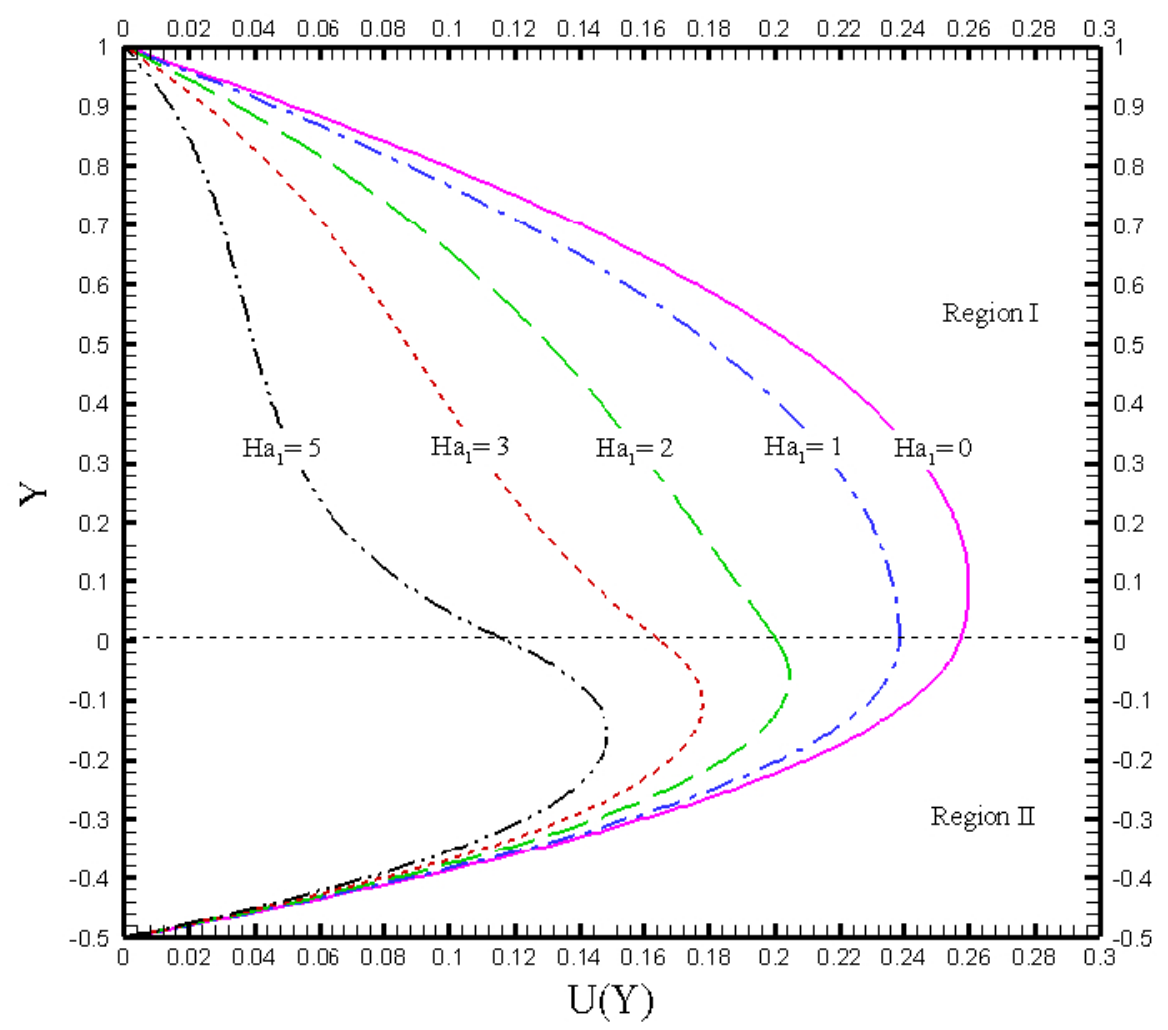

(a) For various values of $\mathrm{Ha} 1$ with $\mathrm{Ha} 2=1$ 


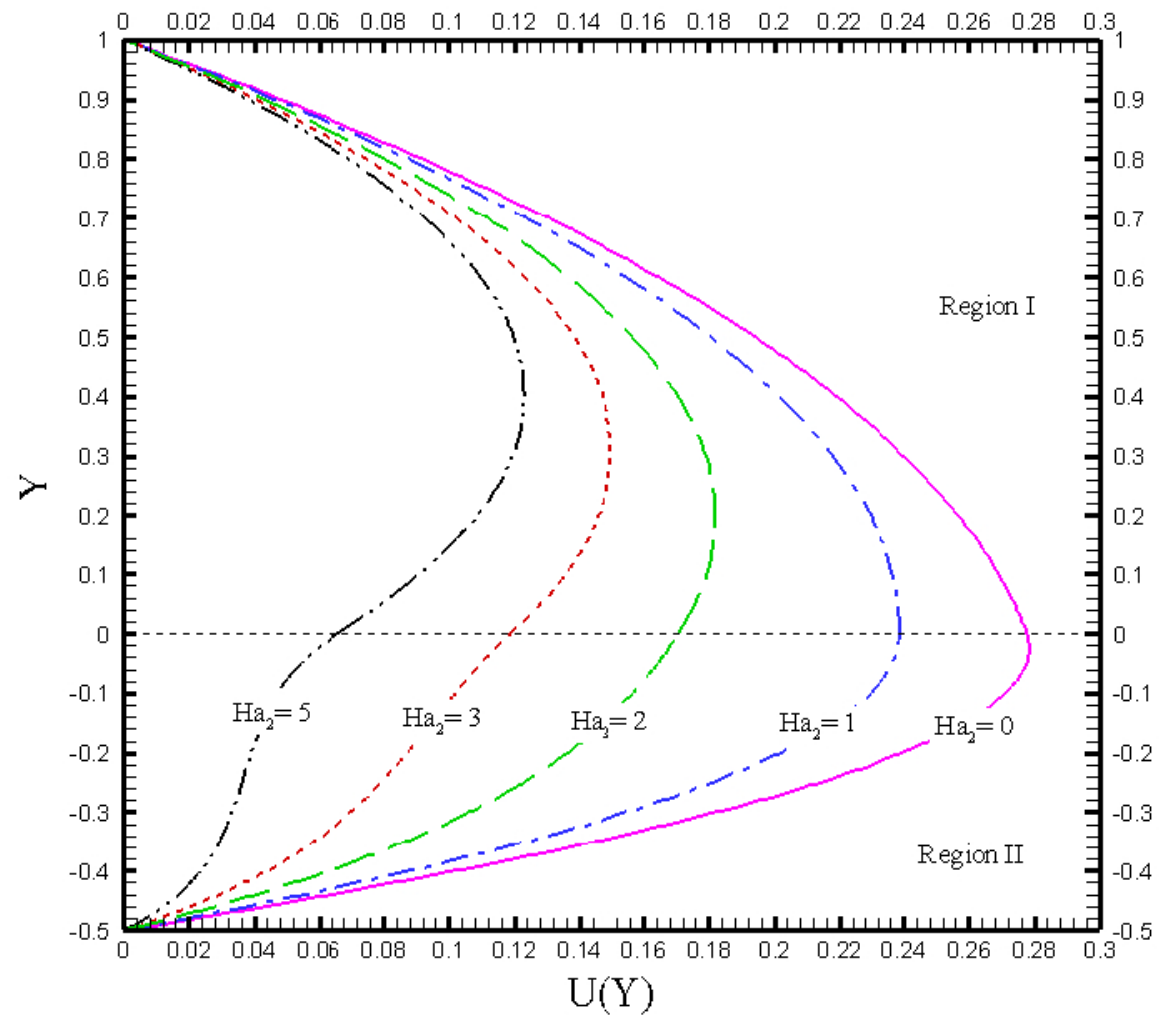

(b) For various values of $\mathrm{Ha} 2$ with $\mathrm{Ha} 1=1$

Fig. 6 The dimensionless velocity profiles $U(Y)$ in the case of $k 1=k 2=1$ and $h=2$. 


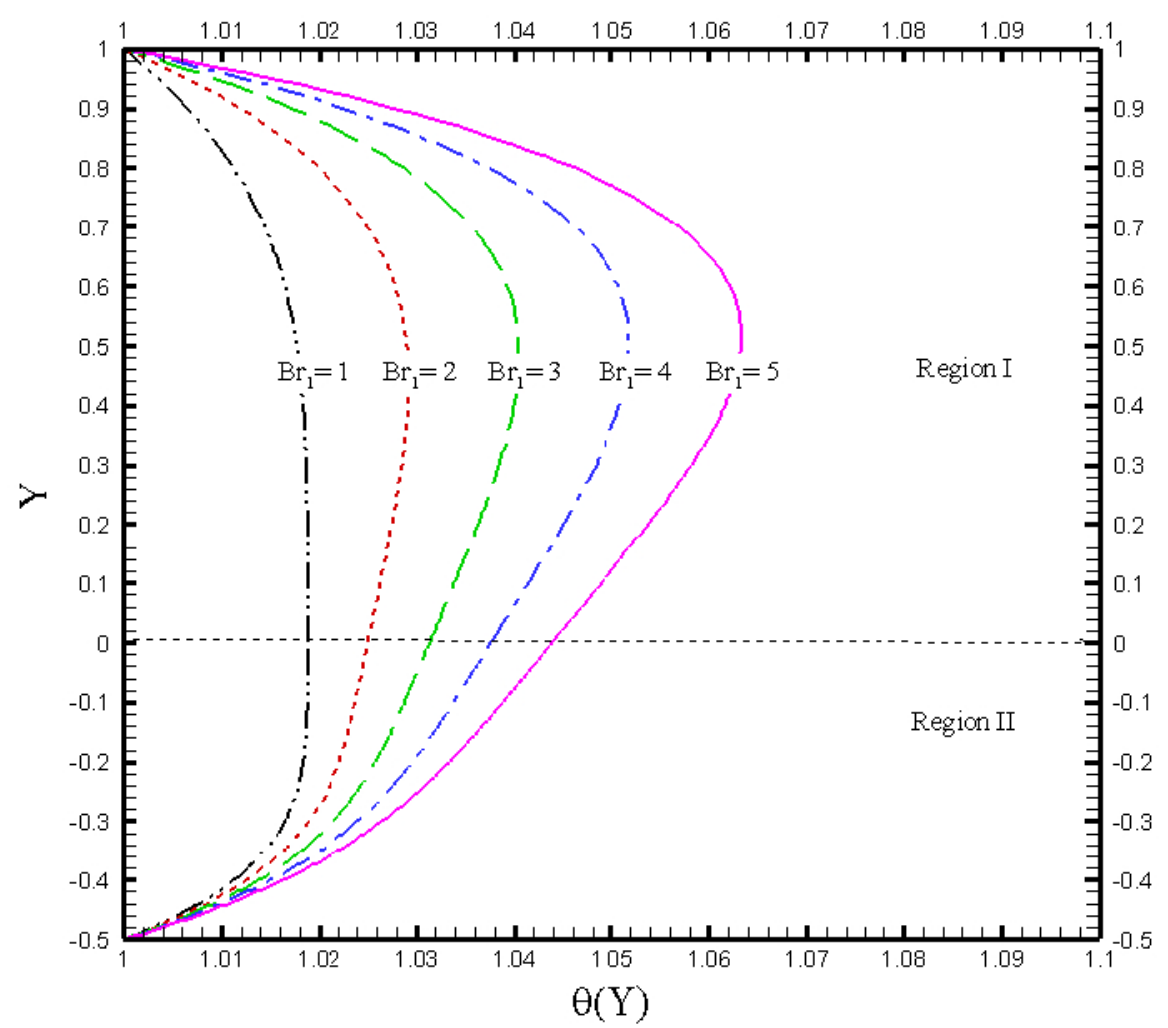

(a) For various values of $\mathrm{Br} 1$ with $\mathrm{Br} 2=1$ 


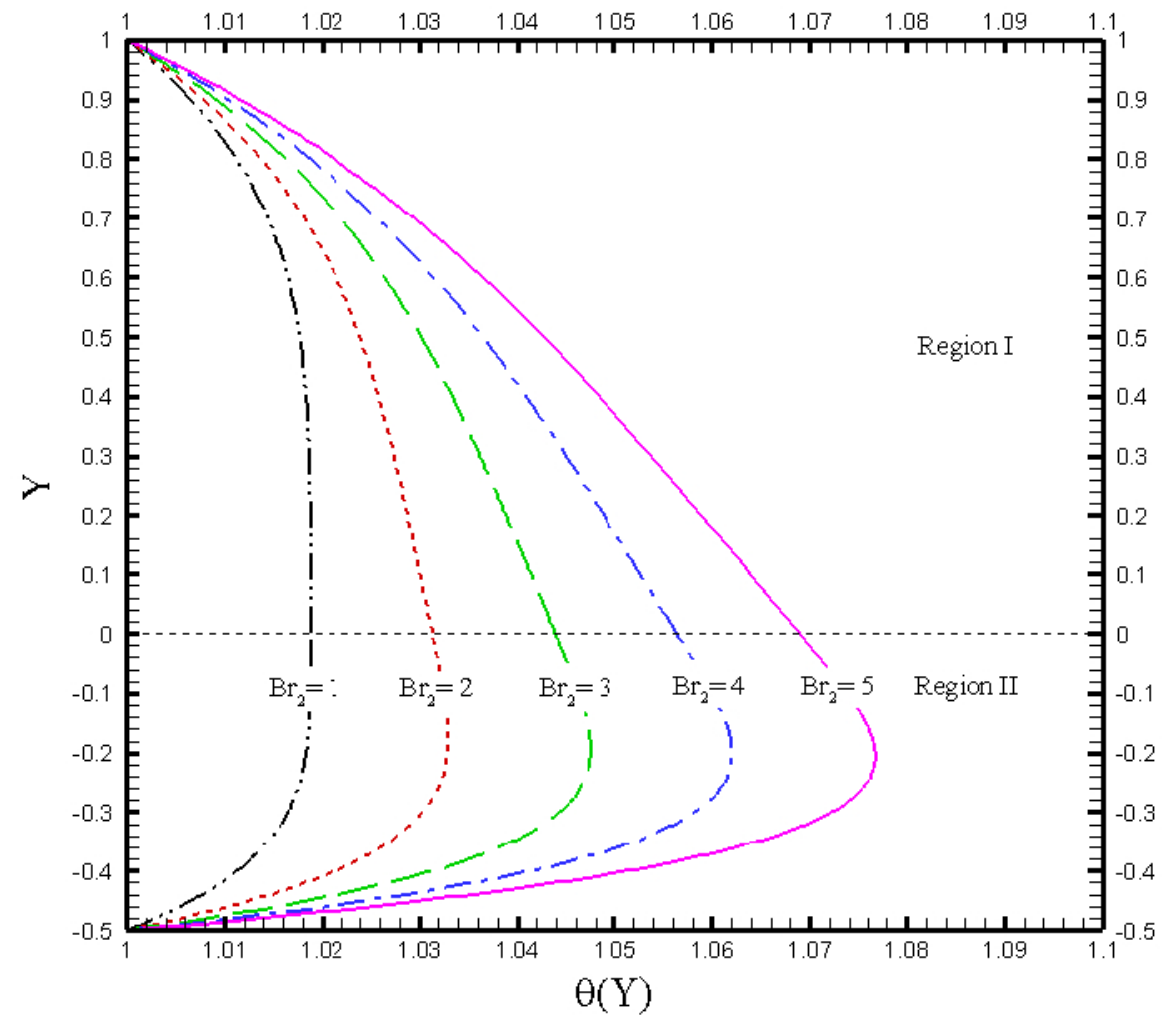

(b) For various values of with

Fig. 7 The dimensionless temperature profiles $\theta(Y)$ in the case of $k 1=k 2=1, \mathrm{Ha} 1=\mathrm{Ha} 2=1, \mathrm{Nt}=\mathrm{Nb}=0.1$ and $h=2$ 


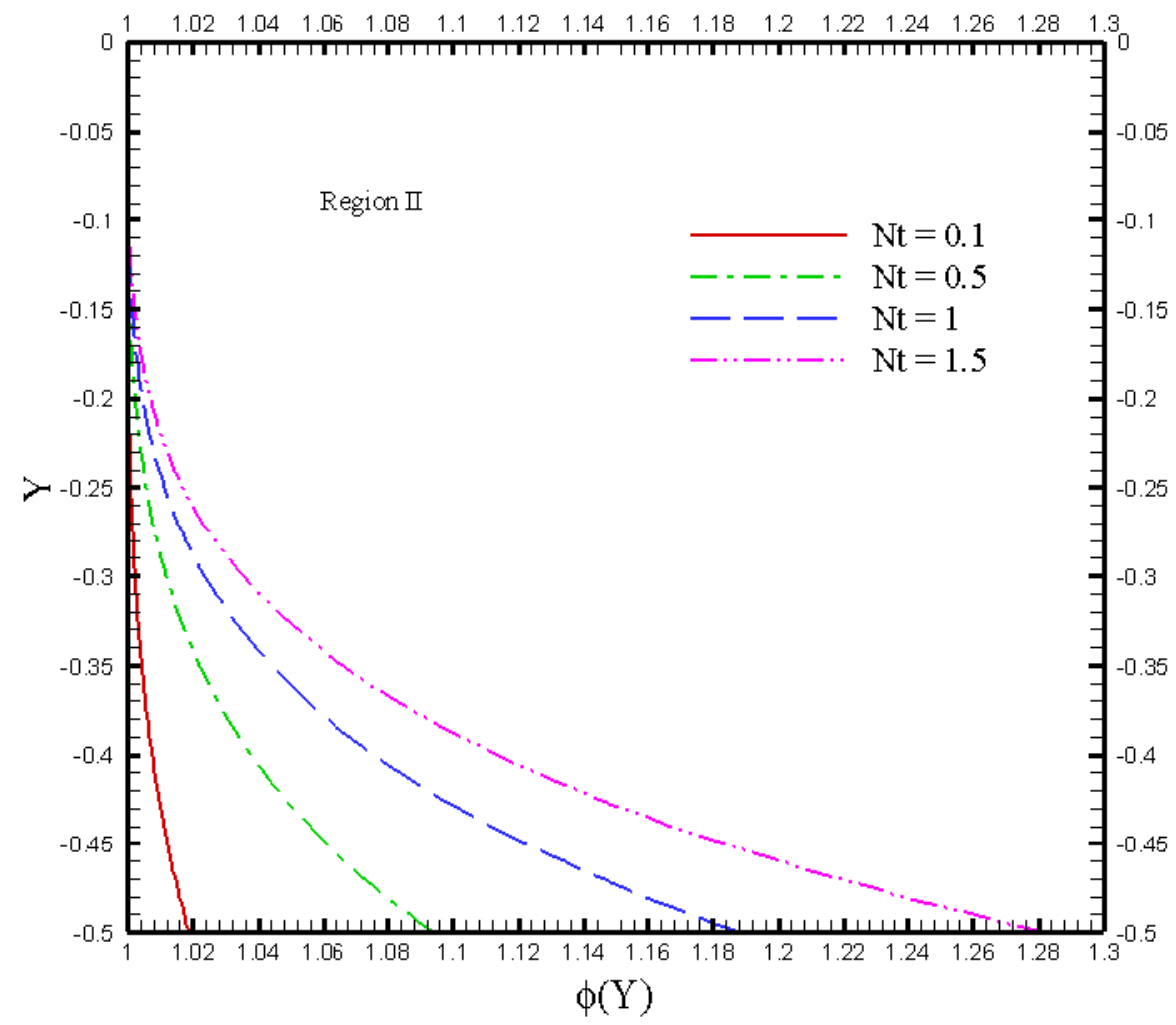

Fig.8 The nanoparticle volume fraction profiles for various values of $\Phi(Y)$ in the case of $k 1=k 2=1$, $\mathrm{Ha} 1=\mathrm{Ha} 2=1, \mathrm{Nb}=0.1, \mathrm{Br} 1=\mathrm{Br} 2=1$ and $\mathrm{h}=2$. 
Fig. 9 Variation of the local Sherwood number with the thermophoresis parameter Nt for various values of $\mathrm{Nb}$ in the case of $\mathrm{k} 1=\mathrm{k} 2=1, \mathrm{Ha} 1=\mathrm{Ha} 2=1, \mathrm{Nb}=0.1, \mathrm{Br} 1=\mathrm{Br} 2=1$ and $\mathrm{h}=2$. 


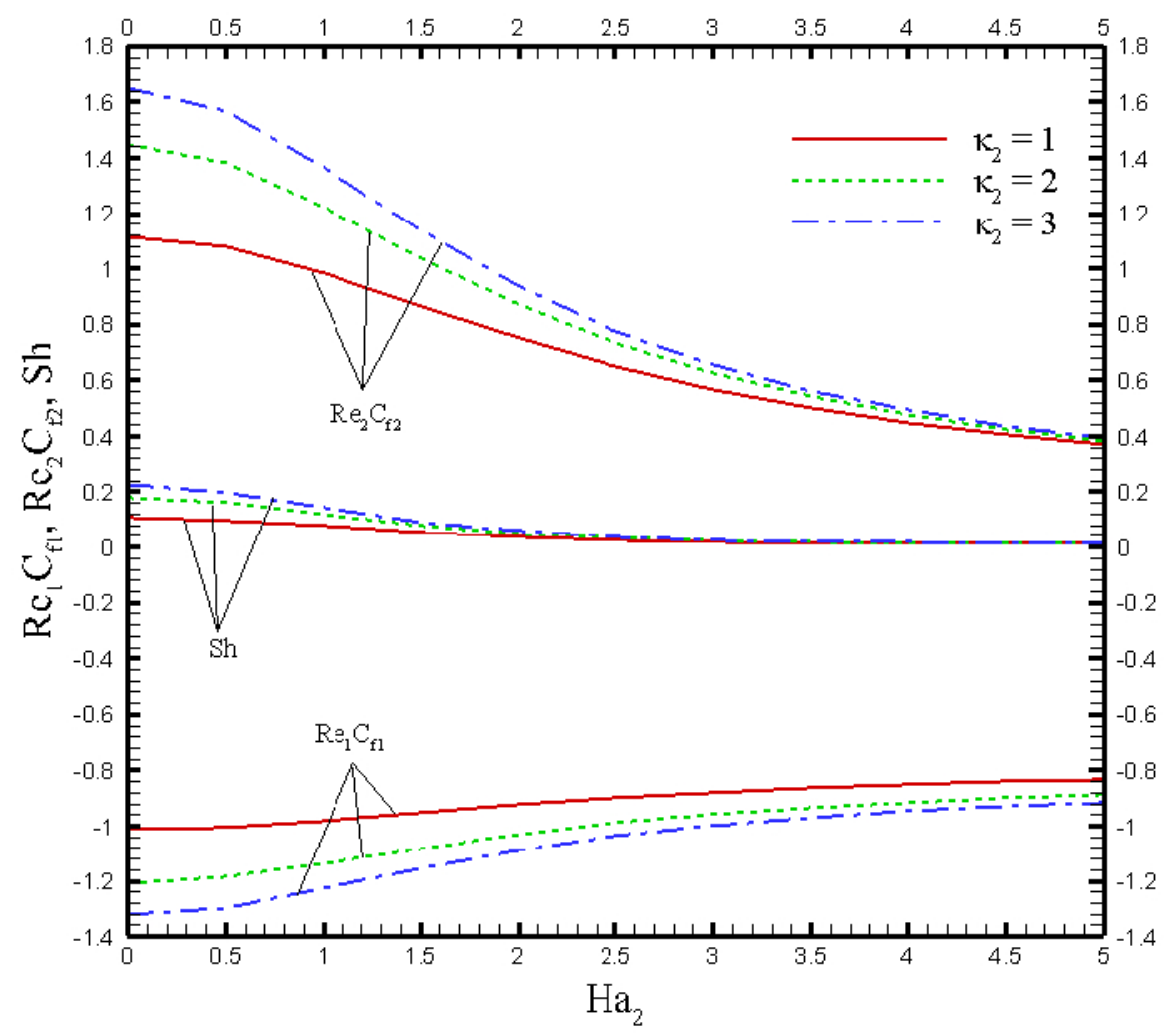

Fig.10 Variation of local skin friction and the local Sherwood number with Ha2 for various values of k2 in the case of $\mathrm{k} 1=1, \mathrm{Ha} 1=1, \mathrm{Nt}=\mathrm{Nb}=0.1, \mathrm{Br} 1=\mathrm{Br} 2=1$ and $\mathrm{h}=2$. 


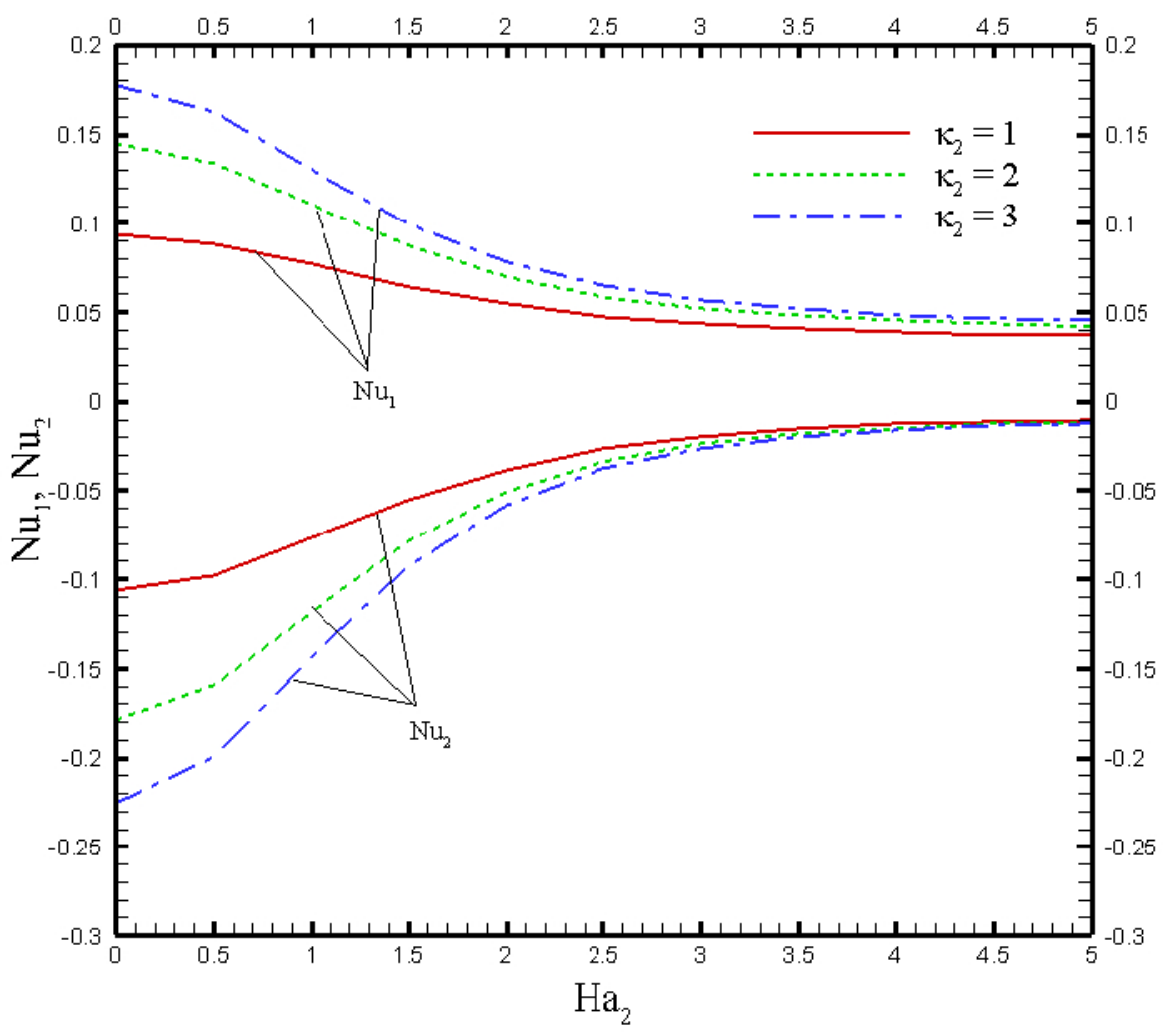

Fig.11 Variation of the local Nusselt number with $\mathrm{Ha} 2$ for various values of $\mathrm{k} 2$ in the case of $\mathrm{k} 1=1, \mathrm{Ha} 1=1$, $\mathrm{Nt}=\mathrm{Nb}=0.1, \mathrm{Br} 1=\mathrm{Br} 2=1$ and $\mathrm{h}=2$. 


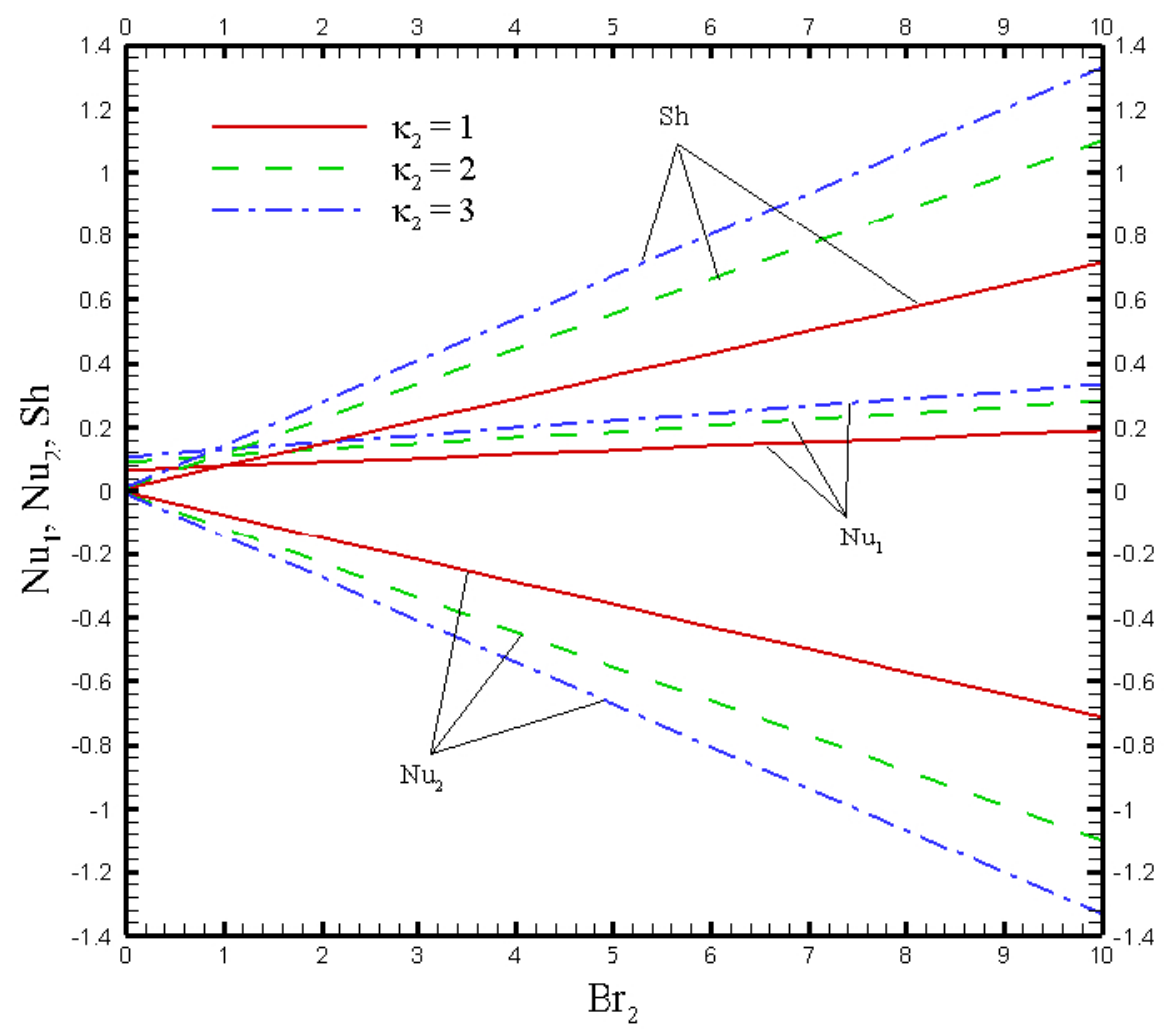

Fig.12 Variation of the local Nusselt number and the local Sherwood number with $\mathrm{Br} 2$ for various values of $\mathrm{k} 2$ in the case of $\mathrm{k} 1=1, \mathrm{Br} 1=1, \mathrm{Nt}=\mathrm{Nb}=0.1, \mathrm{Ha} 1=\mathrm{Ha} 2=1$ and $\mathrm{h}=2$. 
Table I. The maximum error $E(m)$ for different $\kappa_{1}$ in the case of $\kappa_{2}=1, H a_{1}=H a_{2}=1$, $B r_{1}=B r_{2}=1$ and $N b=N t=0.1$.

\begin{tabular}{|c|c|c|c|c|c|}
\hline$E(m)$ & \multicolumn{5}{|c|}{$\kappa_{2}=1$} \\
\hline Order & $\kappa_{1}=1$ & $\kappa_{1}=2$ & $\kappa_{1}=3$ & $\kappa_{1}=5$ & $\kappa_{1}=10$ \\
\hline$m=10$ & 0.0391 & 0.0049 & 0.0064 & 0.3116 & 113.111 \\
\hline$m=20$ & $1.736 \times 10^{-5}$ & $8.908 \times 10^{-9}$ & $1.205 \times 10^{-8}$ & $4.050 \times 10^{-7}$ & $2.250 \times 10^{-5}$ \\
\hline$m=30$ & $6.248 \times 10^{-9}$ & $1.358 \times 10^{-13}$ & $7.908 \times 10^{-13}$ & $6.312 \times 10^{-10}$ & $3.683 \times 10^{-7}$ \\
\hline$m=40$ & $6.351 \times 10^{-12}$ & $4.957 \times 10^{-18}$ & $3.267 \times 10^{-17}$ & $1.919 \times 10^{-12}$ & $7.055 \times 10^{-8}$ \\
\hline
\end{tabular}


Table II. The maximum error $E(m)$ for different $\kappa_{2}$ in the case of $\kappa_{1}=1, H a_{1}=H a_{2}=1$, $B r_{1}=B r_{2}=1$ and $N b=N t=0.1$.

\begin{tabular}{|c|c|c|c|c|c|}
\hline$E(m)$ & \multicolumn{5}{|c|}{$\kappa_{1}=1$} \\
\hline Order & $\kappa_{2}=1$ & $\kappa_{2}=2$ & $\kappa_{2}=3$ & $\kappa_{2}=5$ & $\kappa_{2}=10$ \\
\hline$m=10$ & 0.0391 & $3.671 \times 10^{-4}$ & 0.0044 & 0.0079 & 531.073 \\
\hline$m=20$ & $1.736 \times 10^{-5}$ & $1.966 \times 10^{-8}$ & $1.469 \times 10^{-6}$ & $4.438 \times 10^{-7}$ & $8.150 \times 10^{-7}$ \\
\hline$m=30$ & $6.248 \times 10^{-9}$ & $4.219 \times 10^{-13}$ & $3.612 \times 10^{-10}$ & $3.521 \times 10^{-9}$ & $2.561 \times 10^{-7}$ \\
\hline$m=40$ & $6.351 \times 10^{-12}$ & $1.570 \times 10^{-17}$ & $9.436 \times 10^{-14}$ & $2.516 \times 10^{-11}$ & $1.630 \times 10^{-8}$ \\
\hline
\end{tabular}

